\title{
Egocentric and Allocentric Spatial Memory in Mild Cognitive Impairment with Real-World and Virtual Navigation Tasks: A Systematic Review
}

\author{
Cosimo Tuena $^{\mathrm{a}, *}$, Valentina Mancuso ${ }^{\mathrm{a}, 1}$, Chiara Stramba-Badiale $^{\mathrm{a}, 1}$, Elisa Pedroli $^{\mathrm{a}, \mathrm{b}}$, \\ Marco Stramba-Badiale ${ }^{\mathrm{c}}$, Giuseppe Riva ${ }^{\mathrm{a}, \mathrm{d}}$ and Claudia Repetto ${ }^{\mathrm{e}}$ \\ ${ }^{a}$ Applied Technology for Neuro-Psychology Lab, IRCCS Istituto Auxologico Italiano, Milan, Italy \\ ${ }^{\mathrm{b}}$ Faculty of Psychology, Universitá eCampus, Novedrate, Italy \\ ${ }^{\mathrm{c}}$ Department of Geriatrics and Cardiovascular Medicine, IRCCS Istituto Auxologico Italiano, Milan, Italy \\ ${ }^{\mathrm{d}}$ Humane Technology Lab, Universitá Cattolica del Sacro Cuore, Milan, Italy \\ ${ }^{\mathrm{e}}$ Department of Psychology, Universitá Cattolica del Sacro Cuore, Milan, Italy
}

Accepted 12 October 2020

\begin{abstract}
.
Background: Spatial navigation is the ability to estimate one's position on the basis of environmental and self-motion cues. Spatial memory is the cognitive substrate underlying navigation and relies on two different reference frames: egocentric and allocentric. These spatial frames are prone to decline with aging and impairment is even more pronounced in Alzheimer's disease (AD) or in mild cognitive impairment (MCI).

Objective: To conduct a systematic review of experimental studies investigating which MCI population and tasks are used to evaluate spatial memory and how allocentric and egocentric deficits are impaired in MCI after navigation.

Methods: PRISMA and PICO guidelines were applied to carry out the systematic search. Down and Black checklist was used to assess methodological quality.

Results: Our results showed that amnestic MCI and AD pathology are the most investigated typologies; both egocentric and allocentric memory are impaired in MCI individuals, and MCI due to AD biomarkers has specific encoding and retrieval impairments; secondly, spatial navigation is principally investigated with the hidden goal task (virtual and real-world version), and among studies involving virtual reality, the privileged setting consists of non-immersive technology; thirdly, despite subtle differences, real-world and virtual versions showed good overlap for the assessment of MCI spatial memory.

Conclusion: Considering that MCI is a subclinical entity with potential risk for conversion to dementia, investigating spatial memory deficits with navigation tasks might be crucial to make accurate diagnosis and rehabilitation.
\end{abstract}

Keywords: Alzheimer's disease, embodiment, mild cognitive impairment, navigation, spatial memory, virtual reality

\section{INTRODUCTION}

Exploring, orienting, and navigating would not be possible without a spatial representation of the

\footnotetext{
${ }^{1}$ These authors contributed equally to this work.

*Correspondence to: Cosimo Tuena, Applied Technology for Neuro-Psychology Lab, IRCCS Istituto Auxologico Italiano, via Magnasco 2, 20149, Milan, Italy. Tel.: +39 3902 619112726;

E-mail: c.tuena@auxologico.it.
}

environment. Spatial navigation is the ability to estimate one's position on the basis of both static environmental (allothetic) and dynamic (idiothetic) self-motion cues [1]. While the former is based on visual perception and comprises stable objects, such as landmarks, the latter is based on vestibular, proprioceptive, interoceptive, and motor commands and comprises body-based (i.e., path integration and spatial updating) self-motion [1-3]. Both cues are 
integrated to build up spatial representations that determine one's position and orientation within the environment [3, 4]. In this regard, both cognitive and bodily inputs yield to a successful navigation $[4,5]$. Navigation relies on spatial memory that encompasses processes such as encoding, storing, recognizing and recalling spatial information about the environment, the objects and agents within it [6-8]. Considering that spatial memory is the cognitive substrate underlying navigation, its study is crucial to comprehend and investigate mechanisms and processing that determine our behavior within the environment. Spatial memory depends on two different reference representations that organize the information coming from the environment: egocentric and allocentric frames [9, 10]. On one hand, the egocentric frame refers to the individual location in the environment and it is based on subject-to-object relations. In fact, this memory subsystem employs the self as the reference frame in order to create a body-centered representation. On the other hand, the allocentric frame refers to object-to-object relations independently of individual's location in the environment. This memory subsystem employs the object and/or the environment as the reference frame in order to create a world-centered representation. To successfully navigate within the environment, it is necessary to flexibly switch and combine these two reference frames according to environmental needs [1]. Studies underlying neural mechanism of spatial memory date back to the second half of the 19th century. Egocentric and allocentric frames exhibit separate neural circuits mediating different spatial strategies [11-13]. Concerning allocentric frame, O'Keefe and Dostrovsky [14] found that specific hippocampal cells, called place cells, create a cognitive map of the space, firing when a rat occupies a particular location in the environment. These findings were consistent also in humans $[15,16]$. Other important cells in the medial temporal lobe, grid cells [17], head direction cells [18], and boundary cells [19], accomplish a successful cognitive map and navigation, by providing information to the hippocampus. Other neural substrates which are typically responsible for allocentric processing are parahippocampal and retrosplenial cortex (RSC) [20, 21]. With respect to egocentric frame, parietal area and dorsal striatum are responsible of the egocentric visual representations of the space, which depend both on visuospatial processing and on individual's position, actions and decisions in proximity to discrete landmarks [10, 22, 23]. The ability to switch from one frame to the other involves the posterior cingulate cortex (PCC) and the RSC instead [13, 24, 25]. Furthermore, egocentric and allocentric frames share some common networks like the bilateral fronto-temporal regions [26-28]. Hence, spatial memory encoding is a bottom-up process that starts in the parietal 'window' (a visual egocentric representation of the space) and goes through medial parietal and temporal regions for storage and vice versa for spatial memory recall [13], where frontal regions play a broader role in memory processing, spatial attention and egocentric/allocentric strategy selection $[28,29]$.

\section{Spatial memory decline in mild cognitive impairment}

Navigation and spatial memory are prone to deterioration with normal aging [30, 31]. Frames switching abilities and allocentric difficulties have been reported in healthy older people [32]. Indeed, older individuals tend to prefer egocentric compared to allocentric navigation strategies [33]. In addition, self-motion appears to be compromised in aging [31, 34]. These impairments reflect neurophysiological alternations that occur in regions which are crucial for spatial cognition such as medial temporal areas, basal ganglia, and prefrontal cortex [35]. Deterioration is even more pronounced in neurodegenerative diseases like Alzheimer's disease (AD). In fact, due to the gradual degeneration of specific brain areas (i.e., entorhinal, medial temporal, medial parietal cortices) critical in AD diagnosis [36-38], egocentric and allocentric memory are impaired [39]. Specifically, Serino and colleagues [40] reviewed egocentric and allocentric deficits in $\mathrm{AD}$ patients reporting a prevalence of allocentric abilities impairments. Similar deficits [39] have been found also in prodromal AD (amnestic mild cognitive impairment; aMCI) with hippocampal amnesia [41] or in MCI due to AD biomarkers [42].

MCI is considered a transitional period between normal and pathological cognitive aging [43, 44]. It is characterized by a subtle general decline in cognitive functions, usually affecting first memory domain [45]. The objective decline essentially preserves daily activities and is insufficient to constitute dementia [46]. It has been mainly linked to AD progression [45]. Indeed, prodromic signs of AD can be detected in aMCI by means of: neuropsychological tests, such as the Free and Cued Selective Reminding Test (FCSRT) [47], which can detect hippocampal (H) and non-hippocampal (NH) amnesia according 
to Dubois and Albert criteria [41]; biomarkers of the National Institute on Aging and Alzheimer's Association Research Framework [38] or Albert's criteria for MCI due to AD [42] to identify neuropathological signs of AD in MCI; genetic profiling according to $A P O E \& 4$ [48] non-carriers (defined with - or homozygous $-1-$ in the papers included in the Results section) or $\varepsilon 4$ carriers (+), more specifically with heterozygous $(+/-)$ and homozygous $(+/+)$ carriers, or according to TOMM40 poly-T length polymorphisms [49] distinguishing individuals with short (S), very long (VL) homozygous and heterozygous (S/VL) profiles at risk for AD. Finally, mixed forms of $\mathrm{AD}$ pathology and cerebrovascular disease (i.e., striatal lacunar lesions/white matter hyperintensities [WMH]) can be evaluated according to Fazekas's criteria [50].

However, the heterogeneity of this clinical condition has prevented to reach a general consensus about its diagnosis. In fact MCI could differ for type of cognitive domain impaired (aMCI versus non amnestic; naMCI), the quantity of domains compromised (single versus multiple domains; abbreviated sd and md hereafter) and the etiology (e.g., neurodegenerative, cerebrovascular, depression/neuropsychiatric) $[45,51,52]$. With respect to spatial navigation, several studies have reported impairments in both allocentric and egocentric frame and in the ability to switch between them [40,53-55] in aMCI. These findings have been supported by neuropathological evidence of neurodegeneration of hippocampal areas and RSC, regions involved in spatial representations $[39,56,57]$. Preliminary findings have been found also in other neurodegenerative diseases, such as dementia with Lewy body [58], frontotemporal dementia [59], Parkinson's disease [60], and in vascular cognitive impairment [61, 62]. Additionally, subjective navigation impairment has been reported also in naMCI and in individuals with subjective cognitive decline [63].

\section{Virtual reality for assessment of spatial memory}

Virtual reality (VR) is a new technology that has been increasingly employed to study human spatial memory since it allows to provide navigation mechanisms analogous to those activated in real-life navigation $[64,65]$. Its potential relies on the capability to adapt and tailor environments according to patient's and task's needs, for example by changing or eliminating landmarks or pathways in spatial memory tasks $[29,65,66]$. Further, via VR it is possible to manipulate user's spatial frame from egocentric to allocentric or vice versa [64, 67-69]. Indeed, several studies have employed VR to investigate spatial memory, reproducing classical paradigms used to study navigation such as the hidden goal task (HGT), a human analogue of the Morris water maze [70]. In the VR HGT participants, by using the mouse, are asked to retrieve goal position in a circular map (the arena) by using both starting position (egocentric) and distal cues (allocentric) or just one of these. The relation between goal, starting point and distal cues was showed before each trial but then according to the subtest (allocentric, egocentric or mixed) cues were removed/left. Additionally, after $30 \mathrm{~min}$ allocentric (delayed) memory was tested. The procedure was the same for the Blue Velvet Arena (the real-world version; BVA) but this time participants walked in a $2.9 \mathrm{~m}$ dark arena with luminous goal locations and egocentric/allocentric cues. Other alternative tasks achieved promising diagnostic results with VR [29, $55,66,71-74]$, and with real-space versus virtual versions [75-77] or only real-world tasks [53, 78-80]. Moreover, VR is one of the preferred tools to study spatial memory since it allows the user to actively navigate within the virtual environments, enhancing spatial and episodic performances [74, 81]. In fact, active navigation in virtual environments involves both bodily and cognitive activities [5] by including motor commands, proprioceptive information, vestibular information, decision-making, allocation of attentional resources, and manipulation of spatial information [4]. Conversely, passive navigation implies only visual information [4].

In this regard, VR is a simulative technology which offers ecological, controlled and standardized environments with different degrees of immersion (i.e., immersive, semi and non-immersive) and interaction (e.g., controllers, treadmill, joypad) [65, 82-84]. Indeed, VR can also be considered embodied thank to its potential to provide interactive and multisensory information [85]. VR mimics the neurocognitive process known as embodiment. During our daily experience, the body matrix, a supramodal multisensory body representation, allows us to correctly predict bodily experiences through top-down paths (predictive coding) and to feel located within our body and in the space around (sense of presence) [86]. In the same way, VR tries to predict simulations by generating the sense of presence in the virtual body and environment [87]. In this sense, virtual embodiment is the result of these two key elements and arises as the sense of controlling, own- 


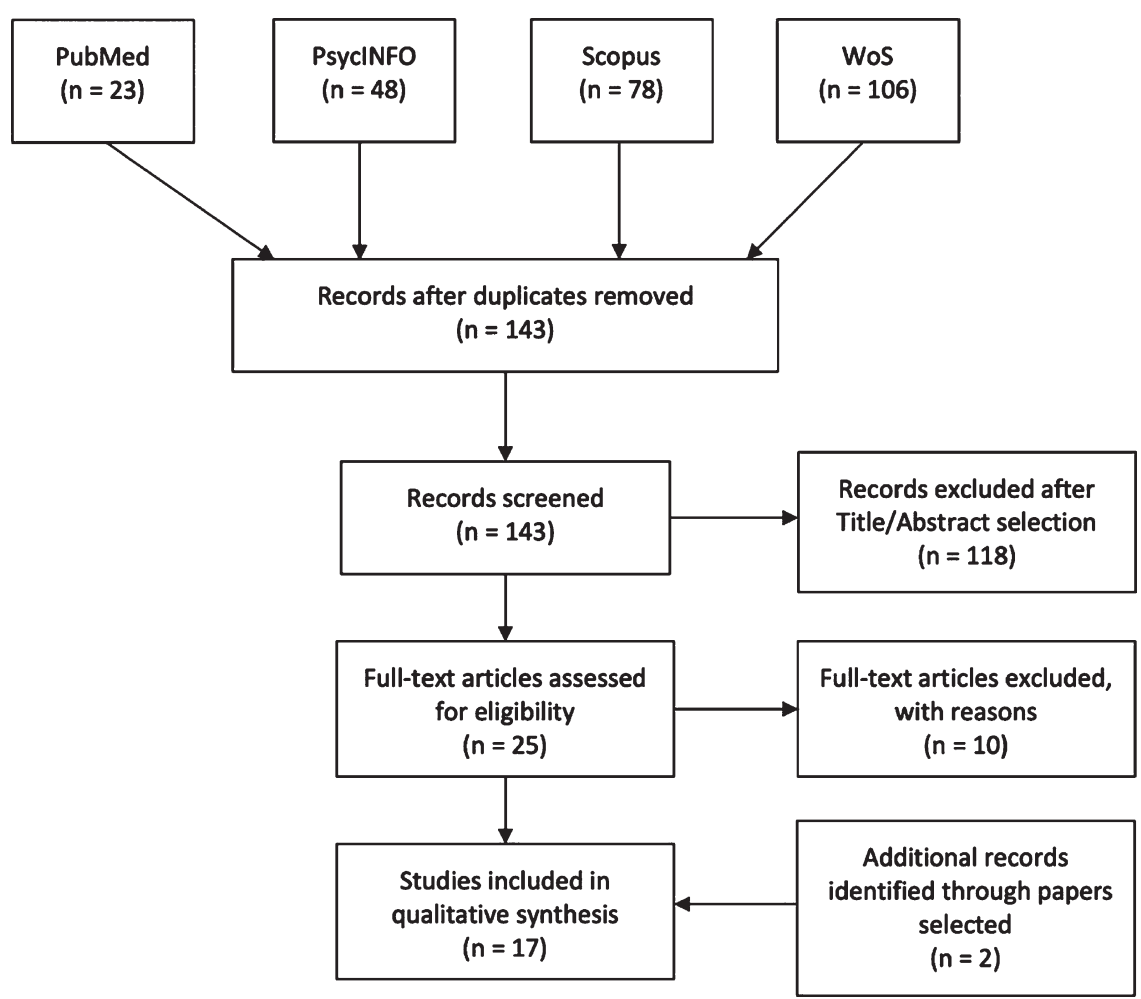

Fig. 1. PRISMA flowchart

ing and feeling located within a virtual body and in the space [88]. In particular, during navigation, effective virtual embodiment is provided by idiothetic and multisensory integration of information coming from internal and external cues [2, 89-91].

To our knowledge there is no systematic review summarizing the deficit of egocentric and allocentric spatial memory in real-world and virtual navigation tasks in MCI. We conducted this review to provide an overview of: 1) egocentric and allocentric spatial memory deficits in MCI profiles; 2) real-world versus virtual navigation performances in MCI.

\section{METHODS}

Preferred Reporting Items for Systematic reviews and Meta-Analysis (PRISMA) guidelines were followed [92].

\section{Search strategy}

Four high-quality databases (PubMed, PsycINFO, Web of Science, Elsevier-Scopus) were used to perform the search on 13 March 2020. Keywords used for each separate string were ("mild cognitive impairment") AND ("egocentric" OR "allocentric" OR "spatial memory") AND ("virtual" OR "real") and were searched through Title/Abstract, Abstract, Topic and Title/Abstract/Keywords respectively for each database. Figure 1 shows the paper selection procedure and number of selected/excluded articles. Two papers $[93,94]$ did not appear in the databases and were added while reading the papers during full-text selection.

\section{Selection criteria}

Studies that uniquely investigated egocentric and allocentric spatial memory in MCI with real or virtual navigation tasks were selected. We excluded articles that did not specifically mention egocentric and allocentric memory by means of navigation tasks; that is where locations/paths where not encoded and retrieved through an act of active navigation $[4,74]$ for egocentric and allocentric memory. Additionally, studies where the full text was not available or where the abstract lacked basic information for review were removed. Non-English papers, reviews, meeting abstracts, conference proceedings, notes, case reports, letters to the editor, research protocols, 
patents, editorials, and other editorial materials were also excluded.

\section{Quality assessment and data abstraction}

PRISMA guidelines were strictly followed; search results found by the first author (CT) were shared with the review co-authors (VM, CS-B) for individual selection of papers in order to reduce bias, and disagreements concerning the type of navigation and spatial memory tested were resolved through consensus. PICO (Population, Intervention, Comparison and Outcome) guidelines were preferred as they provide more inclusive search results [95]. Hence, research question was "How are egocentric and allocentric memory (Outcome) affected in MCI (Population) with real-world and virtual (Intervention) navigation tasks?". Comparison term was set as not applicable because, as emerged in an exploratory search, currently only few studies [75, 76, 94, 96] assess real-world versus virtual navigation in MCI for egocentric and allocentric spatial memory. The risk of bias for each single study was assessed following the Cochrane guidelines [97] by CT, VM, and CS-B (see Supplementary Figure 1); not admitted (N/A) answers were recorded as Cochrane risk of bias is suitable only for experimental designs. Despite we acknowledge that this systematic review does not concern a clinical intervention but the presence-absence of a deficit, Table 1 shows studies design according to definitions of experiment, quasi-experiment $(\mathrm{QE})$ and non-experiment in systematic reviews [98]. Furthermore, all the papers included did not meet the criteria (e.g., randomization) of experimental studies and were considered as QE (see also risk of bias in Supplementary Figure 1); despite control over $\mathrm{QE}$ is less rigorous than experiments, external validity and strength of causal inference is still possible, especially when design-specific assumptions are met (e.g., independence assumption) [98]. In this regard researchers should bear in mind that results of included studies concerning the "effect estimates... are valid at or near the locus on the "natural' process that produced the exogenous variation in exposure" [98]. In order to assess methodological quality (reporting, internal and external validity and power), two authors (VM and CS-B) used the Down and Black checklist for randomized and non-randomized studies [99]. Quality scores are interpreted as excellent (26-28), good (20-25), fair (15-19), and poor $(<14)$ [100] (see Supplementary Figure 2). As some items were strictly applicable for clinical intervention trial N/A items were also recorded: 34 for internal validity (bias), 52 for internal validity (confounding), and 24 for reporting. Average Down and Black scale score for the included studies was $14.17(\mathrm{SD}=2.14)$ without N/A. Data extracted from each included study were as follows: reference, populations included, type of navigation task, study design, neuropsychological/behavioral correlates, neurological correlates and spatial memory findings in MCI.

\section{RESULTS}

Our search identified 17 studies that specifically assessed egocentric and allocentric memory with real-world and virtual navigation tasks in MCI. Findings are shown in Table 1 according to reference, year, sample(s), navigation task, neuropsychological correlates, neural correlates and main spatial memory findings. In particular, we will focus our result section on: 1) egocentric and allocentric memory deficits in MCI subtypes; 2) real-world versus virtual navigation performances of MCI.

\section{Egocentric and allocentric spatial memory in MCI after navigation tasks}

\section{Egocentric and allocentric spatial memory in $a M C I$}

Some studies investigated aMCI without distinguishing sd and md types by using Petersen's criteria $[44,45,101,102]$. In this cluster, egocentric memory recall was more impaired in aMCI than in healthy controls (HC) [72, 106, 107, 109]. Allocentric retrieval was similar among aMCI and $\mathrm{HC}$ in one study [109] but more impaired in other findings [54, 72, 106, 107]. aMCI performed similarly to $\mathrm{HC}$ in the mixed allocentric-egocentric recall in one study [109] but were more impaired in another [107]. aMCI performed better on allocentric recall than $\mathrm{AD}$ in Plancher and colleagues [106]. Interestingly, AD, aMCI, and HC were less impaired in allocentric but not egocentric recall in the active VR exploration than in the passive VR exploration [106]. Similar to $\mathrm{HC}$, egocentric, allocentric, and mixed egocentricallocentric recall performance was more impaired in aMCI than in subjective cognitive decline group [107].

Learning across egocentric task was similar among aMCI and $\mathrm{HC}$ across trials but impaired in the allocentric learning [72]. Interestingly, Weniger and colleagues [72] found that larger volumes of the right- 
Table 1

Summary of the studies analyzed

\begin{tabular}{|c|c|c|c|c|c|c|}
\hline Reference & Samples & Navigation Task & Study design & $\begin{array}{l}\text { Neuropsychological } \\
\text { correlates }\end{array}$ & Brain correlates & Spatial Memory Outcome \\
\hline [96] & $\begin{array}{l}21 \text { mild/moderate AD }(\text { age }=75.8, \\
\mathrm{SD}=1.2 ; \text { edu }=12.4, \mathrm{SD}=0.7 ; \\
\mathrm{MMSE}=23.1, \mathrm{SD}=4) \\
\mathbf{1 1} \text { aMCIsd }(\mathrm{age}=71.7, \mathrm{SD}=2 ; \\
\text { edu }=15.5, \mathrm{SD}=0.7 ; \mathrm{MMSE}=28.6, \\
\mathrm{SD}=1.5) \\
\mathbf{1 8} \text { aMCImd }(\text { age }=72.9, \mathrm{SD}=2.4 ; \\
\text { edu }=13.9, \mathrm{SD}=0.8 ; \mathrm{MMSE}=27.1, \\
\mathrm{SD}=2.3) \\
\mathbf{7} \text { naMCI }(\mathrm{age}=70.6, \mathrm{SD}=3 ; \\
\text { edu }=14.3, \mathrm{SD}=1.1 ; \mathrm{MMSE}=29, \\
\mathrm{SD}=1) \\
\mathbf{8} \mathrm{SMC}(\mathrm{age}=65.6, \mathrm{SD}=4 ; \text { edu }=16.4, \\
\mathrm{SD}=0.6 ; \mathrm{MMSE}=29.8, \mathrm{SD}=0.4) \\
\mathbf{2 6} \mathrm{HC}(\mathrm{age}=69.4, \mathrm{SD}=1.3 ; \text { edu }=15.5, \\
\mathrm{SD}=0.6 ; \mathrm{MMSE}=29.3, \mathrm{SD}=0.9)\end{array}$ & $\begin{array}{l}\text { Real-world: } \\
\text { HGT-MWM } \\
\text { (BVA) } \\
\text { Virtual: HGT- } \\
\text { MWM (2D VR) }\end{array}$ & $\begin{array}{l}\text { QE: } \\
\text { Within-between }\end{array}$ & Not investigated & Not investigated & $\begin{array}{l}\text { AD and aMCImd were impaired in all } \\
\text { subtests of the virtual and real versions } \\
\text { of the HGT. aMCImd had difficulties in } \\
\text { orientation as well as learning and } \\
\text { remembering locations but aMCI only } \\
\text { in remembering. aMCIsd were impaired } \\
\text { in allocentric and real-world navigation. } \\
\text { naMCI and SMC were similar to HC }\end{array}$ \\
\hline [104] & $\begin{array}{l}21 \text { aMCIcom }(\text { age }=62.5, \mathrm{SD}=7.6 \\
\text { edu }=14, \mathrm{SD}=4.1 ; \mathrm{MMSE}=25.7 \\
\mathrm{SD}=3.3) \\
21 \text { aMCI }(\text { age }=68.1, \mathrm{SD}=10.1 ; \\
\text { edu }=14.1, \mathrm{SD}=3.7 ; \mathrm{MMSE}=26.1 \\
\mathrm{SD}=2.9) \\
\mathbf{2 2} \mathrm{HC}(\mathrm{age}=65.8, \mathrm{SD}=6.4 ; \text { edu }=15.8 \\
\mathrm{SD}=2.7 ; \mathrm{MMSE}=29.1, \mathrm{SD}=1.4)\end{array}$ & $\begin{array}{l}\text { Virtual: HGT- } \\
\text { MWM (2D VR) }\end{array}$ & $\begin{array}{l}\text { QE: } \\
\text { Within-between }\end{array}$ & $\begin{array}{l}\text { aMCIcom did not differ } \\
\text { in the egocentric, } \\
\text { allocentric and delayed } \\
\text { allocentric memory } \\
\text { depending on } \\
\text { neuropsychiatric } \\
\text { symptoms (anxiety, } \\
\text { depression, anxiety and } \\
\text { depression, alcohol } \\
\text { abuse) }\end{array}$ & Not investigated & $\begin{array}{l}\text { aMCIcom and aMCI have impaired } \\
\text { spatial memory but aMCIcom has } \\
\text { further egocentric deficit compared to } \\
\text { aMCI }\end{array}$ \\
\hline [108] & $\begin{array}{l}21 \text { mild AD }(\text { age }=75.9, \mathrm{SD}=5.6 ; \\
\text { edu }=12.3, \mathrm{SD}=3.3 ; \text { MMSE score } 23.1 \\
\mathrm{SD}=3.7) \\
\mathbf{1 0} \text { HaMCI }(\text { age }=77.3, \mathrm{SD}=10.8 \\
\text { edu }=13.6, \mathrm{SD}=3.5 ; \mathrm{MMSE}=26.2 \\
\mathrm{SD}=3.0) \\
\mathbf{3 2} \text { NHaMCI }(\text { age }=72.7, \mathrm{SD}=9.2 ; \\
\text { edu }=14.3, \mathrm{SD}=3.3 ; \mathrm{MMSE} \text { score } 27.6 \\
\mathrm{SD}=1.5) \\
\text { naMCI }(\mathrm{age}=72, \mathrm{SD}=7.9 ; \text { edu }=14.7 \\
\mathrm{SD}=2.8 ; \text { MMSE }=27.9, \mathrm{SD}=2.3) \\
\mathbf{2 8} \mathbf{H C}(\mathrm{age}=68.987 .2 ; \text { edu }=15.5 \\
\mathrm{SD}=3.0 ; \text { MMSE score } 29.3, \mathrm{SD}=0.9)\end{array}$ & $\begin{array}{l}\text { Real-world: } \\
\text { HGT-MWM } \\
\text { (BVA) }\end{array}$ & $\begin{array}{l}\text { QE: } \\
\text { Within-between }\end{array}$ & $\begin{array}{l}\text { Memory tests } \\
\text { performance do not } \\
\text { account for spatial } \\
\text { memory deficits in aMCI } \\
\text { types }\end{array}$ & Not investigated & $\begin{array}{l}\text { Egocentric and allocentric spatial } \\
\text { learning and retrieval performances } \\
\text { discriminate between aMCI of } \\
\text { hippocampal and frontal type }\end{array}$ \\
\hline
\end{tabular}


Table 1

Continued

\begin{tabular}{|c|c|c|c|c|c|c|}
\hline Reference & Samples & Navigation Task & Study design & $\begin{array}{l}\text { Neuropsychological } \\
\text { correlates }\end{array}$ & Brain correlates & Spatial Memory Outcome \\
\hline [94] & $\begin{array}{l}21 \text { mild/moderate AD } \\
11 \text { aMCIsd } \\
\text { 31 aMCImd } \\
\text { aMCI further stratified } \\
(\mathrm{HaMCI}=10 ; \mathrm{NHaMCI}=32 ; \\
\text { ApoE4 +=12; ApoE4 }-=30) \\
7 \text { naMCI } \\
\mathbf{2 8} \text { HC }\end{array}$ & $\begin{array}{l}\text { Real-world: } \\
\text { HGT-MWM } \\
\text { (BVA) } \\
\text { Virtual: HGT- } \\
\text { MWM (2D VR) }\end{array}$ & $\begin{array}{l}\text { QE: } \\
\text { Within-between }\end{array}$ & $\begin{array}{l}\text { FCSRT was useful for } \\
\text { differentiating aMCI in } \\
\text { addition to spatial } \\
\text { memory task }\end{array}$ & Not investigated & $\begin{array}{l}\text { Heterogeneous findings were found } \\
\text { in aMCI population. However, } \\
\text { aMCImd, HaMCI and } \\
\text { ApoE } 4+\text { showed similar AD } \\
\text { performances in the Real-world and } \\
\text { virtual subtasks }\end{array}$ \\
\hline [93] & $\begin{array}{l}\mathbf{1 6} \text { mild AD }(\text { age }=75.5, S D=6.1 \\
\text { edu }=12.2, \mathrm{SD}=3.6 ; \mathrm{MMSE}=23.8 \\
\mathrm{SD}=2.8) \\
\mathbf{1 1} \text { aMCI } \mathbf{\varepsilon} 4+(\text { age }=75.5, \mathrm{SD}=8.2 \\
\text { edu }=13.7, \mathrm{SD}=3.6 ; \mathrm{MMSE}=26.7 \\
\mathrm{SD}=3.2) \\
\mathbf{2 3} \mathrm{aMCI} \varepsilon \mathbf{\varepsilon}-(\mathrm{age}=74, \mathrm{SD}=11 ; \\
\text { edu }=14.5, \mathrm{SD}=3.3 ; \mathrm{MMSE}=27.7 \\
\mathrm{SD}=1.5) \\
\mathbf{2 8} \mathbf{H C}(\mathrm{age}=68.9, \mathrm{SD}=7.3 ; \\
\text { edu }=15.5, \mathrm{SD}=3 ; \mathrm{MMSE}=29.3 \\
\mathrm{SD}=0.9)\end{array}$ & $\begin{array}{l}\text { Real-world: } \\
\text { HGT-MWM } \\
\text { (BVA) }\end{array}$ & $\begin{array}{l}\text { QE: } \\
\text { Within-between }\end{array}$ & $\begin{array}{l}\text { Auditory-verbal learning } \\
\text { scores in aMCI groups } \\
\text { did not affect spatial } \\
\text { memory performances }\end{array}$ & Not investigated & $\begin{array}{l}\text { aMCI } \varepsilon 4+\text { had lower spatial } \\
\text { memory performances compared to } \\
\text { HC and aMCI } \varepsilon 4 \text { - groups and } \\
\text { similar deficits to AD patients }\end{array}$ \\
\hline [75] & Same as [108] without naMCI & $\begin{array}{l}\text { Virtual: } \\
\text { HGT-MWM (2D } \\
\text { VR) with spatial } \\
\text { memory } \\
\text { correlations of } \\
\text { the real-world } \\
\text { version [108] }\end{array}$ & $\begin{array}{l}\text { QE: } \\
\text { Within-between }\end{array}$ & $\begin{array}{l}\text { FCSRT is able to detect } \\
\text { patients that perform } \\
\text { worst on spatial } \\
\text { navigation learning and } \\
\text { recall }\end{array}$ & Not investigated & $\begin{array}{l}\text { Real and virtual versions of the } \\
\text { HGT showed high correlations } \\
\text { across spatial memory results. In the } \\
\text { virtual version HaMCI showed } \\
\text { greater impairments compared to } \\
\text { NHaMCI similar to AD }\end{array}$ \\
\hline [71] & $\begin{array}{l}\mathbf{3 3} \text { aMCI } \varepsilon \mathbf{4}-/-(\text { age }=74.4, \\
\mathrm{SD}=10.8, \text { edu }=13.4, \mathrm{SD}=2.4, \\
\mathrm{MMSE}=27, \mathrm{SD}=2.1) \\
\mathbf{2 6} \mathrm{aMCI} \mathbf{\varepsilon}+/-(\mathrm{age}=74.9, \\
\mathrm{SD}=7.3, \mathrm{edu}=13.6, \mathrm{SD}=2.9, \\
\mathrm{MMSE}=26.7, \mathrm{SD}=2.6) \\
\mathbf{1 5} \mathrm{aMCI} \boldsymbol{\mathbf { 4 }}+/+(\mathrm{age}=71.7 \\
\mathrm{SD}=7.3, \mathrm{edu}=12.7, \mathrm{SD}=3, \\
\mathrm{MMSE}=25.5, \mathrm{SD}=2.4)\end{array}$ & $\begin{array}{l}\text { Virtual: HGT- } \\
\text { MWM (2D VR) }\end{array}$ & $\begin{array}{l}\text { QE: } \\
\text { Within-between }\end{array}$ & $\begin{array}{l}\text { Verbal and visual } \\
\text { memory performances } \\
\text { did not change the main } \\
\text { effects for group in all } \\
\text { spatial navigation } \\
\text { subtasks }\end{array}$ & $\begin{array}{l}\text { Right Hp volume } \\
\text { accounted for } 50 \% \text { of the } \\
\text { association between } \\
\text { being a } \varepsilon 4+/+ \text { carrier (as } \\
\text { opposed to } \varepsilon 4-/- \text { carrier) } \\
\text { and spatial navigation on } \\
\text { the allocentric subtask, } \\
69 \% \text { of the association on } \\
\text { the delayed subtask, and } \\
4 \% \text { of the association on } \\
\text { the egocentric subtask. } \\
\text { No spatial learning } \\
\text { correlation was found }\end{array}$ & $\begin{array}{l}\text { APOE } \varepsilon 4 \text { carriers exhibited poorer } \\
\text { overall navigation accuracy. } \\
\text { Specifically, APOE } \varepsilon 4 \text { homozygotes } \\
\text { performed worse than } \\
\text { heterozygotes. These results were at } \\
\text { least due to right hippocampal } \\
\text { volume differences }\end{array}$ \\
\hline
\end{tabular}




\begin{tabular}{|c|c|c|c|c|c|c|}
\hline Reference & Samples & Navigation Task & Study design & $\begin{array}{l}\text { Neuropsychological } \\
\text { correlates }\end{array}$ & Brain correlates & Spatial Memory Outcome \\
\hline [53] & $\begin{array}{l}59 \text { aMCI APOE } \varepsilon 3 \text { homozygotes with } \\
\text { TOMM } 40 \text { " } 523 " \text { polymorphism divided } \\
\text { in: } \\
\mathbf{1 6} \text { aMCI S/S }(\text { age }=73.9 ; \mathrm{SD}=6.0 ; \\
\text { edu }=14.8 ; \text { MMSE }=27.6, \mathrm{SD}=2.4) \\
\mathbf{2 8} \text { aMCI } \mathbf{S} / \mathrm{VL}(\mathrm{age}=75.4, \mathrm{SD}=6.2 ; \\
\text { edu }=15.1, \mathrm{SD}=3.1 ; \mathrm{MMSE}=26.2, \\
\mathrm{SD}=2.7) \\
\mathbf{1 5} \mathbf{a M C I} \mathbf{V L} / \mathrm{VL}(\mathrm{age}=75.4, \mathrm{SD}=6.5, \\
\text { edu }=13.6, \mathrm{SD}=2.6 ; \mathrm{MMSE}=27.6 \\
\mathrm{SD}=2.8)\end{array}$ & $\begin{array}{l}\text { Real-world: } \\
\text { HGT-MWM } \\
\text { (BVA) }\end{array}$ & $\begin{array}{l}\text { QE: } \\
\text { Within-between }\end{array}$ & Not investigated & $\begin{array}{l}\text { Extent of errors } \\
\text { on the egocentric } \\
\text { task correlated } \\
\text { negatively with } \\
\text { IPP and PCC } \\
\text { thickness. Extent } \\
\text { of errors in the } \\
\text { allocentric task } \\
\text { correlated } \\
\text { negatively with } \\
\text { Hp volumes and } \\
\text { entorhinal } \\
\text { cortical thickness }\end{array}$ & $\begin{array}{l}\text { Greater influence of TOMM } 40 \text { " } 523 \text { " } \\
\text { polymorphism on spatial navigation } \\
\text { performance was observed in allocentric } \\
\text { compared to egocentric memory. } \\
\text { In particular S/VL and VL/VL had } \\
\text { poorer allocentric than egocentric } \\
\text { navigation compared with the S/S }\end{array}$ \\
\hline [110] & $\begin{array}{l}55 \text { aMCI } \\
53 \mathrm{HC}\end{array}$ & $\begin{array}{l}\text { Real-world: } \\
\text { HGT-MWM } \\
\text { (BVA) }\end{array}$ & $\begin{array}{l}\text { QE: } \\
\text { Within-between }\end{array}$ & $\begin{array}{l}\text { In aMCI executive } \\
\text { functioning was } \\
\text { associated with } \\
\text { allocentric memory. } \\
\text { Trend for association } \\
\text { between visuospatial } \\
\text { domain and allocentric } \\
\text { navigation. Verbal } \\
\text { memory was associated } \\
\text { with egocentric } \\
\text { navigation. In HC } \\
\text { cognitive functions were } \\
\text { not associated with } \\
\text { allocentric or egocentric } \\
\text { navigation }\end{array}$ & $\begin{array}{l}\text { In HC Hp } \\
\text { volumes were not } \\
\text { associated with } \\
\text { either } \\
\text { performance on } \\
\text { spatial navigation } \\
\text { or memory tests } \\
\text { In aMCI both Hp } \\
\text { volumes were } \\
\text { associated with } \\
\text { allocentric } \\
\text { navigation }\end{array}$ & $\begin{array}{l}\text { Allocentric and egocentric memory } \\
\text { loaded highly onto the same single } \\
\text { factor ("Spatial navigation") and } \\
\text { showed low loadings on other factors in } \\
\text { both CN and aMCI groups }\end{array}$ \\
\hline [55] & $\begin{array}{l}20 \text { mild AD }(\text { age }=73.65, \mathrm{SD}=2.476 \\
\text { edu }=11.25, \mathrm{SD}=2.51 ; \mathrm{MMSE}=19.3, \\
\mathrm{SD}=1) \\
\mathbf{3 0} \text { aMCIsd }(\text { age }=70, \mathrm{SD}=1.68 ; \\
\text { edu }=12.96, \mathrm{SD}=2.34 ; \mathrm{MMSE}=27.03, \\
\mathrm{SD}=0.8) \\
\text { 30 aMCImd }(70.06, \mathrm{SD}=1.63 ; \\
\text { edu }=12.03, \mathrm{SD}=2.32 ; \\
\mathrm{MMSE}=26.167, \mathrm{SD}=0.91) \\
\mathbf{3 0} \mathbf{H C}(\mathrm{age}=69.86, \mathrm{SD}=1.43 ; \\
\text { edu }=13.13, \mathrm{SD}=2.72 ; \mathrm{MMSE}=28.06, \\
\mathrm{SD}=1)\end{array}$ & $\begin{array}{l}\text { Virtual: 2D } \\
\text { VRNT }\end{array}$ & $\begin{array}{l}\text { QE: } \\
\text { Within-between }\end{array}$ & $\begin{array}{l}\text { Auditory-verbal memory } \\
\text { positively correlated with } \\
\text { correct responses in } \\
\text { egocentric and } \\
\text { allocentric memory and } \\
\text { negatively correlated } \\
\text { with the time spent to } \\
\text { find the given goal in the } \\
\text { both egocentric and } \\
\text { allocentric tasks }\end{array}$ & Not investigated & $\begin{array}{l}\text { aMCI groups (md and sd) had more } \\
\text { impaired egocentric compared to } \\
\text { allocentric memory. AD and aMCImd } \\
\text { were more impaired egocentric and } \\
\text { allocentric memory compared to } \\
\text { aMCIsd and HC }\end{array}$ \\
\hline
\end{tabular}


Table 1

Continued

\begin{tabular}{|c|c|c|c|c|c|c|}
\hline Reference & Samples & Navigation Task & Study design & $\begin{array}{l}\text { Neuropsychological } \\
\text { correlates }\end{array}$ & Brain correlates & Spatial Memory Outcome \\
\hline [107] & $\begin{array}{l}29 \text { aMCI }(\text { age }=74.62, S D=7.87 \\
\text { edu }=14.83, S D=3.49 ; \mathrm{MMSE}=26.17, \\
\mathrm{SD}=2.55) \\
46 \mathrm{SCD}(\mathrm{age}=69.09, \mathrm{SD}=6.44 \\
\mathrm{edu}=15.22, \mathrm{SD}=3.25 ; \mathrm{MMSE}=28.5 \\
\mathrm{SD}=1.53) \\
\mathbf{1 7} \mathrm{HC}(\mathrm{age}=71.59, \mathrm{SD}=5.65 \\
\mathrm{edu}=16.47, \mathrm{SD}=3.39 ; \mathrm{MMSE}=29.41 \\
\mathrm{SD}=0.80)\end{array}$ & $\begin{array}{l}\text { Real-world: } \\
\text { HGT-MWM } \\
\text { (BVA) }\end{array}$ & $\begin{array}{l}\text { QE: } \\
\text { Within-between }\end{array}$ & $\begin{array}{l}\text { Distance error in all the } \\
\text { subtests correlated } \\
\text { negatively with the } \\
\text { MMSE }\end{array}$ & $\begin{array}{l}\text { Hcy level was } \\
\text { related to worse } \\
\text { mixed egocentric- } \\
\text { allocentric } \\
\text { performance in } \\
\text { the aMCI group, } \\
\text { white matter } \\
\text { lesions did not } \\
\text { affect spatial } \\
\text { memory }\end{array}$ & $\begin{array}{l}\text { Higher plasma Hcy level was related to } \\
\text { worse mixed egocentric-allocentric and } \\
\text { egocentric navigation performance, but } \\
\text { not to worse allocentric navigation } \\
\text { performance, independently of Fazekas } \\
\text { scores }\end{array}$ \\
\hline \multirow[t]{2}{*}{ [76] } & $\begin{array}{l}21 \text { mild } \mathrm{AD}(\mathrm{age}=73.24, \mathrm{SD}=6.93 ; \\
\text { edu }=14.33, \mathrm{SD}=3.74 ; \mathrm{MMSE}=22.33, \\
\mathrm{SD}=2.69) \\
\mathbf{2 8} \text { aMCI due to } \mathrm{AD}(\text { age }=74.46 \\
\mathrm{SD}=5.87 ; \text { edu }=15.39, \mathrm{SD}=2.94 ; \\
\mathrm{MMSE}=27, \mathrm{SD}=2.16) \\
\mathbf{2 8} \mathrm{HC}(\text { ages }=67.50, \mathrm{SD}=7.13 ; \\
\text { edu }=16.65, \mathrm{SD}=2.32 ; \mathrm{MMSE}=29.55 \\
\mathrm{SD}=0.69)\end{array}$ & $\begin{array}{l}\text { Virtual: vYSA } \\
\text { (2D VR) } \\
\text { Real-world: } \\
\text { HGT-MWM } \\
\text { (BVA) }\end{array}$ & $\begin{array}{l}\text { QE: } \\
\text { Within-between }\end{array}$ & Not investigated & $\begin{array}{l}\text { Lower total Hp } \\
\text { and BF1, BF6 } \\
\text { volumes } \\
\text { correlated with } \\
\text { less accurate } \\
\text { allocentric } \\
\text { performance. }\end{array}$ & $\begin{array}{l}\text { aMCI group preferred the egocentric } \\
\text { strategy. Participants in the aMCI group } \\
\text { who preferred the egocentric strategy } \\
\text { had less accurate performance in the } \\
\text { allocentric task than those who } \\
\text { preferred the allocentric strategy }\end{array}$ \\
\hline & & & & & $\begin{array}{l}\text { In aMCI right Hp } \\
\text { volume } \\
\text { accounted for } \\
22 \%, \text { whereas } \\
\text { total and left Hp } \\
\text { volumes } \\
\text { accounted for } \\
14 \% \text { and } 9 \% \text { of } \\
\text { the association } \\
\text { between strategy } \\
\text { preference and } \\
\text { performance in } \\
\text { the allocentric } \\
\text { task }\end{array}$ & \\
\hline [106] & $\begin{array}{l}15 \text { AD }(\text { age }=76.53, \mathrm{SD}=5.51 ; \\
\text { edu }=3.2, \mathrm{SD}=1.5 ; \mathrm{MMSE}=19.3, \\
\mathrm{SD}=3.5) \\
\mathbf{1 5} \text { aMCI }(\text { age }=78.06, \mathrm{SD}=5.76 \\
\text { edu }=5, \mathrm{SD}=1.7 ; \mathrm{MMSE}=26.5 \\
\mathrm{SD}=1.6) \\
\mathbf{2 1} \mathrm{HC}(\mathrm{age}=76.95, \mathrm{SD}=5.8 ; \text { edu }=3.5 \\
\mathrm{SD}=1.5 ; \mathrm{MMSE}=28.7, \mathrm{SD}=0.8)\end{array}$ & $\begin{array}{l}\text { Virtual: } \\
\text { Semi-immersive } \\
\text { active versus } \\
\text { passive } \\
\text { navigation task }\end{array}$ & $\begin{array}{l}\text { QE: } \\
\text { Within-between }\end{array}$ & Not investigated & Not investigated & $\begin{array}{l}\text { aMCI, AD patients were impaired for } \\
\text { both the immediate and delayed recall } \\
\text { of egocentric memory. AD, aMCI } \\
\text { allocentric memory was more impaired } \\
\text { than HC. Active exploration led to lesser } \\
\text { impairment in allocentric recall for all } \\
\text { groups }\end{array}$ \\
\hline
\end{tabular}


Table 1

Continued

\begin{tabular}{|c|c|c|c|c|c|c|}
\hline Reference & Samples & Navigation Task & Study design & $\begin{array}{l}\text { Neuropsychological } \\
\text { correlates }\end{array}$ & Brain correlates & Spatial Memory Outcome \\
\hline [109] & $\begin{array}{l}33 \text { MCI }(\text { age }=64.3, \mathrm{SD}=11.1 ; \\
\text { edu }=11.6, \mathrm{SD}=4.2 ; \mathrm{MMSE}=26.2 \\
\mathrm{SD}=2.7) \\
\mathbf{2 1} \mathbf{H C}(\mathrm{age}=70.7, \mathrm{SD}=10.9 \\
\text { edu }=15.2, \mathrm{SD}=2.3 ; \mathrm{MMSE}=29 \\
\mathrm{SD}=1)\end{array}$ & $\begin{array}{l}\text { Virtual: HGT- } \\
\text { MWM (2D VR) }\end{array}$ & $\begin{array}{l}\text { QE: } \\
\text { Within-between }\end{array}$ & Not investigated & $\begin{array}{l}\text { Any significant } \\
\text { correlations } \\
\text { between spatial } \\
\text { navigation } \\
\text { performance and } \\
\text { rs-fMRI were } \\
\text { found in MCI } \\
\text { patients }\end{array}$ & $\begin{array}{l}\text { MCI have impaired egocentric memory } \\
\text { compared to HC. Allocentric and mixed } \\
\text { egocentric-allocentric memory was } \\
\text { similar among MCI and HC }\end{array}$ \\
\hline [78] & $\begin{array}{l}\mathbf{2 1} \text { aMCI }(\text { age }=70.1, \mathrm{SD}=4.3 \\
\text { edu }=10.3, \mathrm{SD}=1.6) \text { divided in } 11 \\
\mathrm{~A}+\text { and } 10 \mathrm{~A}- \\
\mathbf{1 5} \mathbf{H C}(\text { age }=67.2, \mathrm{SD}=3.8 ; \text { edu }=11.5 \\
\mathrm{SD}=2.1)\end{array}$ & $\begin{array}{l}\text { Real-world } \\
\text { navigation } \\
\text { paradigm }\end{array}$ & $\begin{array}{l}\text { QE: } \\
\text { Within-between }\end{array}$ & $\begin{array}{l}\text { Egocentric and } \\
\text { allocentric memory } \\
\text { differentiate more } \\
\text { accurately A + and A- } \\
\text { than word-list delayed } \\
\text { recall, TMT-B or MMSE }\end{array}$ & $\begin{array}{l}\text { aMCI with lower } \\
\text { neuropsychologi- } \\
\text { cal profile had } \\
\text { higher pontine } \\
\text { tegmentum } \\
\text { rCGM and lower } \\
\text { in right anterior } \\
\text { Hp and left } \\
\text { posterior paraH } \\
\text { during } \\
\text { navigation. } \\
\text { A + had lower } \\
\text { rCGM in the right } \\
\text { anterior Hp, RSC, } \\
\text { Pc and PL during } \\
\text { navigation versus } \\
\text { locomotion } \\
\text { compared to HC. } \\
\text { A + compared to } \\
\text { A- had lower } \\
\text { rCGM in the Hp } \\
\text { and in the right } \\
\text { SPL and } \\
\text { increased in the } \\
\text { pontomedullary } \\
\text { tegmentum. }\end{array}$ & $\begin{array}{l}\text { aMCI have impaired spatial memory } \\
\text { and more spatial visual search compared } \\
\text { to HC. Egocentric and allocentric recall } \\
\text { can differentiate aMCI A + and A-due } \\
\text { to alterations in right Hp, RSC and PL }\end{array}$ \\
\hline
\end{tabular}


Table 1

Continued

\begin{tabular}{|c|c|c|c|c|c|c|}
\hline Reference & Samples & Navigation Task & Study design & $\begin{array}{l}\text { Neuropsychological } \\
\text { correlates }\end{array}$ & Brain correlates & Spatial Memory Outcome \\
\hline [54] & $\begin{array}{l}\mathbf{1 5} \mathrm{AD}(\text { age }=82.93, \mathrm{SD}=5.61 ; \\
\text { edu }=6.60, \mathrm{SD}=3.83 ; \mathrm{MMSE}=23.06, \\
\mathrm{SD}=1.5) \\
\mathbf{1 5} \text { aMCI }(\mathrm{age}=77.53, \mathrm{SD}=5.52 ; \\
\text { edu }=7.73, \mathrm{SD}=4.48 ; \mathrm{MMSE}=22.46, \\
\mathrm{SD}=1.95) \\
\mathbf{1 5} \mathrm{HC}(\mathrm{age}=73.87, \mathrm{SD}=7.38 ; \\
\text { edu }=12.27, \mathrm{SD}=3.88 ; \mathrm{MMSE}=27.52, \\
\mathrm{SD}=1.48)\end{array}$ & $\begin{array}{l}\text { Virtual: } 2 \mathrm{D} \\
\text { navigation task }\end{array}$ & $\begin{array}{l}\text { QE: } \\
\text { Within-between }\end{array}$ & $\begin{array}{l}\text { Egocentric mental } \\
\text { rotation positively } \\
\text { correlated with better } \\
\text { allocentric abilities }\end{array}$ & Not investigated & $\begin{array}{l}\text { aMCI showed deficit in encoding and } \\
\text { storing an allocentric viewpoint } \\
\text { independent representation. aMCI and } \\
\text { AD have impaired allocentric memory } \\
\text { compared to HC }\end{array}$ \\
\hline [72] & $\begin{array}{l}29 \text { aMCI }(\text { age }=59, \mathrm{SD}=9 ; \text { edu }=9.9, \\
\mathrm{SD}=2.0 ; \text { MMSE }=28, \mathrm{SD}=2) \\
\text { composed of } 22 \text { aMCIsd and } 7 \text { aMCImd } \\
29 \mathrm{HC}(\text { age }=59, \mathrm{SD}=8 ; \text { edu }=9.6, \\
\mathrm{SD}=1.7 ; \text { MMSE not reported })\end{array}$ & $\begin{array}{l}\text { Virtual: 2D } \\
\text { Virtual Maze } \\
\text { (egocentric) and } \\
\text { Park (allocentric) }\end{array}$ & $\begin{array}{l}\text { QE: } \\
\text { Within-between }\end{array}$ & $\begin{array}{l}\text { When controlling for } \\
\text { depression and } \\
\text { visuospatial deficits, } \\
\text { group differences } \\
\text { survived. However, for } \\
\text { egocentric memory } \\
\text { depression affected } \\
\text { learning by group } \\
\text { significance }\end{array}$ & $\begin{array}{l}\text { HC, aMCI with } \\
\text { larger volumes of } \\
\text { the right-sided Pc } \\
\text { had better } \\
\text { performances on } \\
\text { egocentric } \\
\text { memory } \\
\text { HC, aMCI with } \\
\text { striatal lacunar } \\
\text { lesions } \\
\text { committed more } \\
\text { errors than } \\
\text { participants } \\
\text { without striatal } \\
\text { lacunar lesions on } \\
\text { egocentric but not } \\
\text { on allocentric } \\
\text { memory }\end{array}$ & $\begin{array}{l}\text { aMCI have impaired allocentric and } \\
\text { egocentric memory. Egocentric spatial } \\
\text { learning was similar among aMCI and } \\
\text { HC. Allocentric spatial learning was } \\
\text { worse in aMCI }\end{array}$ \\
\hline
\end{tabular}

A-, amyloid negative; A+, amyloid positive; AD, Alzheimer's disease; aMCI, amnestic MCI; aMCImd, aMCI multiple domain; aMCIcom, aMCI with neuropsychiatric comorbidity; aMCIsd, aMCI single domain; APOE, apolipoprotein E; ApoE4+, positive APOE isoform E4; ApoE4 -, negative APOE isoform E4; BF1, basal forebrain - posterior part of the Nucleus basalis of Meynert; BF6, basal forebrain - the medial septal nuclei and vertical limb of the diagonal band of Broca; BVA, Blue Velvet Arena; edu, education; FCSRT, Free and Cued Selective Reminding Test; H, hippocampal; HC, healthy controls; HGT, Hidden Goal Task; Hp, hippocampus; IPP, inferior posterior parietal; MCI, mild cognitive impairment; MMSE, Mini-Mental State Examination; MWM,

Morris Water Maze; naMCI, non-amnestic MCI; NH, non-hippocampal; Pc, precuneus; PCC, posterior cingulate cortex; PL, parietal lobe; QE, quasi-experiment; rCGM, regional cerebral glucose metabolism; RSC, retrosplenial cortex; S/S, homozygous for Short poly-T homopolymer; S/VL, heterozygous Short/Very long poly-T homopolymer; SCD, subjective cognitive decline; SMC, subjective memory complaints; SPL, superior parietal lobe; TMT, Trial Making Test; VRNT, Virtual Reality Navigation Task; VL/VL, homozygous for Very Long poly-T homopolymer; VR, virtual reality; vYSA, virtual Y-maze strategy assessment; $\varepsilon 3$, allele $3 ; \varepsilon 4+/-, A P O E$ genotype- $\varepsilon 4$ heterozygous carriers; $\varepsilon 4-/-, A P O E$ genotype- $\varepsilon 4$ non-carriers; $\varepsilon 4+/+, A P O E$ genotype- $\varepsilon 4$ homozygous carriers 
sided precuneus significantly predicted better performance on the egocentric memory task in both aMCI and HC. The authors also found that participants in both groups who had striatal vascular lesions committed more errors than participants without striatal lesions in egocentric memory task, while WMH did not affect egocentric or allocentric memory.

Interestingly, Keynejad and co-authors [104] showed that aMCI individuals with neuropsychiatric comorbidity (com-aMCI) were more impaired on egocentric memory recall compared to aMCI and HC. Both aMCI groups were equally impaired and more impaired than $\mathrm{HC}$ for the allocentric and delayed retrieval. In Weniger and colleagues [72], egocentric and allocentric recall performances between aMCI and $\mathrm{HC}$ were not accounted by spatial cognition test and depressive symptoms. However, egocentric learning (trials) by group interaction did not survive with these variables as covariates.

Conversely for neuropsychiatric symptoms, Keynejad and co-authors [104] found that com-aMCI subgroups (anxiety disorder versus depression, depression and anxiety or alcohol misuse) were equally impaired for all the subtasks of the HGT.

Subjective memory complaints and naMCI groups performed as well as the control group in all the subtests of the versions [96, 108]. Finally, aMCI versus naMCI spatial memory performances were not reported in one study [108].

\section{Egocentric and allocentric spatial memory in aMCI with single and multiple domain}

Another cluster of studies investigated the deficits according to sd and md typologies [44, 45, 101, 102]. In the real-world and VR version Hort and colleagues [96] found that $\mathrm{AD}$ and aMCImd were more impaired than the $\mathrm{HC}$ in all the recall subtests. aMCIsd performed worse than the $\mathrm{HC}$ in the allocentric and delayed recall of the real and virtual versions but as $\mathrm{HC}$ in the other subtests $[94,96]$. The aMCIsd performed as the AD group in the delayed recall of the real and computer version but in all the other recall subtests they performed better [96]. Similarly, Mohammadi and co-authors [55] showed that egocentric and allocentric retrieval was more impaired in mild $\mathrm{AD}$ and aMCImd patients than in aMCIsd and $\mathrm{HC}$ [55]; moreover, egocentric recall was more impaired in aMCIsd, aMCImd, and mild AD than allocentric memory retrieval.

Learning abilities of the aMCImd was similar to AD group, expect for the virtual mixed allocentricegocentric task [96]. aMCIsd had similar learning effect to $\mathrm{HC}$ for the allocentric VR (last trials but not first trial), egocentric real/VR, and allocentricegocentric real task [96].

\section{Egocentric and allocentric spatial memory in aMCI with hippocampal and non-hippocampal amnesia}

Here we discuss the findings of navigation performances of individuals with $\mathrm{H}$ and $\mathrm{NH}$ amnesia detected by means of the FCSRT [41]. Consistently, egocentric, allocentric, and delayed allocentric recall were more impaired in HaMCI than NHaMCI [75, 94, 108]. Mixed egocentric-allocentric memory retrieval was more impaired in HaMCI than NHaMCI in two studies [75, 94] but not in one research [108]. HaMCI was as impaired as mild probable AD for the egocentric, allocentric, and delayed recall but outperformed $\mathrm{AD}$ for the mixed recall [75], but in two studies [94, 108] HaMCI performed equally to AD in all the tasks. NHaMCI outperformed mild AD patients in all the spatial recall tasks in one work [75] but in other findings [94, 108] delayed test was as impaired as mild AD patients. Both HaMCI and NHaMCI were more impaired than controls for all the four HGT recall measures, with NHaMCI having less severe deficits [75, 94, 108].

Learning across allocentric task was more impaired in HaMCI than NHaMCI [75, 108], whereas contrast results were found for egocentric learning $[75,108]$. Mixed learning performance was better for NHaMCI than HaMCI [108]. In particular, learning HaMCI ability was worse than control group and similar to mild AD individuals [108].

\section{Egocentric and allocentric spatial memory in aMCI with $A D$ biomarkers}

Concerning biomarkers of $\mathrm{AD}$ in MCI, studies mainly used genetic markers [48, 49]. aMCI $\varepsilon 4+$ had lower spatial recall performances in all the tests except for the delayed task compared to $\varepsilon 4$ - group $[93,94]$. aMCI $\varepsilon 4+$ group had lower spatial recall performance in all the tests compared to HC [93]. aMCI $\varepsilon 4-$ group showed similar retrieval performances to controls for the egocentric and mixed recall but were outperformed in the allocentric and delayed recall [93]. aMCI $\varepsilon 4+$ showed similar deficits to AD individuals [93, 94], whereas aMCI $\varepsilon 4-$ outperformed $\mathrm{AD}$ group in all the subtests in one study [93] but not in allocentric and delayed in another [94]. 
More specifically, in $\varepsilon 4$ profiles, egocentric, allocentric, and delayed recall were more impaired in aMCI $\varepsilon 4+/+,+/-$ than in the aMCI $\varepsilon 4-/-$ groups [71]. Further, egocentric retrieval was more impaired in the aMCI $\varepsilon 4+/+$ than in the aMCI $\varepsilon 4+/$ - groups. In allocentric and delayed recall, aMCI $\varepsilon 4+/+$ and aMCI $\varepsilon 4+$ /- were equally impaired [71].

According to TOMM40 gene profiling, Laczó and colleagues [53] found that allocentric and delayed recall were more impaired in the aMCI S/VL, VL/VL than in the aMCI S/S groups. Egocentric and mixed egocentric-allocentric memory retrieval were equally impaired in aMCI S/VL, VL/VL, S/S groups. Interestingly, there were not any differences in the $\varepsilon 4$ and TOMM40 profiles in the learning performances in all the subtests $[53,71,93]$. Only one used amyloid pathology [38], and in this study [78], both amyloid positive $(\mathrm{A}+)$ and negative $(\mathrm{A}-)$ aMCI groups showed poorer navigation compared to controls (few items found); in A + group, errors were associated with both egocentric and allocentric recall deficits, whereas in A- group, only with allocentric deficit. Interestingly, aMCI due to AD [42] group preferred egocentric strategy but, overall, strategy preference did not affect allocentric navigation [76]. Those individuals who preferred the egocentric strategy had less accurate performance in the allocentric navigation task than those who preferred the allocentric strategy [76].

\section{Egocentric and allocentric spatial memory in MCI in real-world versus virtual navigation tasks}

In this section we will report MCI performances in the two navigation types. Only few studies compared real-world versus virtual navigation task $[75,94,96]$. In Hort and colleagues [96], recall subtests performances of aMCIsd and aMCImd (aMCImd more impaired than aMCIsd) were equal in both real-world and virtual versions. For learning performances in the first trial of the virtual mixed egocentric-allocentric was similar to control in aMCImd reflecting shortterm visual memory rather than long-term spatial memory. The aMCIsd group was equally impaired in the virtual and real-world allocentric in the first and last trials of task reflecting hippocampal involvement in both versions. The pattern of allocentric memory deficit in aMCImd and aMCIsd was evident with the two navigation tasks. Similarly, in the study by Laczó and collaborators [94], aMCIsd and aMCImd performed in the same way in the two navigation tasks and with the same allocen- tric impaired pattern. They found also that ApoE4 non-carriers had better egocentric memory performances than ApoE4 carriers, which performed as non-carriers in all the other subtests. However, learning trials were not analyzed for the groups. The aMCImd group did better than AD group only in the mixed allocentric-egocentric VR version and in the egocentric and allocentric real-world recall [96], but in Laczó and collagues [94], aMCImd had similar performances to $\mathrm{AD}$ individuals in all the recall tasks. Finally, naMCI performed as HC in both versions in some studies [94, 96]. Interestingly, in Laczó and collaborators [75], correlations between real-world and virtual versions were reported and showed good correlations (mixed egocentric-allocentric: $r=0.76$, $p<0.001$; egocentric: $\mathrm{r}=0.82, p<0.001$; allocentric: $\mathrm{r}=0.83, p<0.001$; delayed: $\mathrm{r}=0.65, p<0.001$ ). They found the same pattern in recall performances between HaMCI and NHaMCI (equal allocentric and delayed performances but HaMCI showed greater impairment for egocentric and mixed task) and between $\mathrm{H} / \mathrm{NH}$ versus $\mathrm{HC}$ in the two versions. This pattern was also confirmed in Laczó and collaborators [94]. Learning performances were more impaired in the real-version for HaMCI in all subtests [108] but in the virtual was equal for egocentric memory in HaMCI and NHaMCI [75]. Parizkova and coauthors [76] used a virtual version of the Y-maze to detect $\mathrm{MCI}$ due to $\mathrm{AD}$ with allocentric or egocentric strategy and study the impact on real-world navigation. They found that aMCI due to AD individuals who preferred egocentric strategy (67\%) compared to those who preferred an allocentric (33\%) navigation in the virtual task had less accurate real-world allocentric memory. This pattern detected with VR reflects $\mathrm{AD}$ pathology that affects medial temporal lobes and leads individuals to adopt compensatory strategies to overcome allocentric deficits. The rest of the studies did not compare real versus virtual versions; hence we provided a qualitative overview of intra-MCI subtypes differences in Table 2.

\section{DISCUSSION}

In the present paper we reviewed studies that assessed egocentric and allocentric spatial memory in MCI, by means of real-world and virtual navigation tasks. According to the sections, our work can be summarized into the following points: 1) aMCI was the most studied subtype as critical prognostic entity for $\mathrm{AD}$; in particular, aMCI is characterized by spa- 


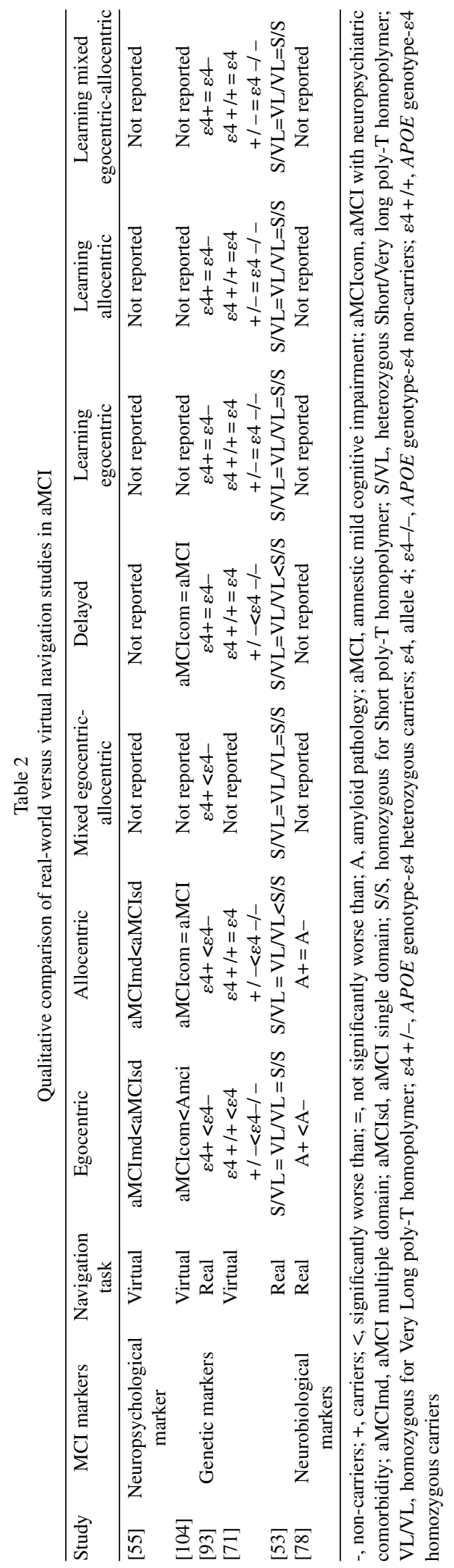

tial memory impairments and, regardless the aMCI subtype, deficits are not only confined to allocentric memory, but extend also to egocentric abilities; moreover, neuropsychological and genetic profiles show specific spatial memory patterns potentially useful to improve diagnosis; 2 ) the preferred navigation task is the 2D VR HGT or the HGT in the BVA; 3 ) real or virtual navigation tasks can be equally sensitive to detect navigation deficits in MCI. To our best knowledge this is the first systematic review that evaluates egocentric and allocentric memory impairments in MCI sample. Our findings could be used to plan future studies to improve diagnosis and prognosis of MCI. More, the review could be used as a starting point to design adequate and innovative rehabilitative solutions for MCI by means of VR thanks to its multisensory, interactive, ecological, standardized, adaptable, and safe potential for active navigation tasks in aging. Findings should be taken with caution as we found poor methodological quality due to the use of $\mathrm{QE}$; however, as the included studies are not concerned with clinical interventions bur rather are interested in detecting a deficit in clinical populations, we must acknowledge that part of this bias is not due to lack of experimental rigor but rather in the design its self of the studies, which could be hardly carried out with a different method.

MCI is an heterogenous diagnostic entity in its neuropsychological and neuropathological profiles and has been initially observed in patients with memory impairments at higher risk of developing AD [51]. Indeed, in our review the most studied subtype was aMCI. This group was categorized by the use of neuropsychological marker (i.e., FCSRT) [41] or with $\mathrm{AD}$ biomarker, such as the presence of positive amyloid pathology or individuals at genetic risk of $A D$ $[38,42,48,49]$. Despite the crucial role of aMCI in $\mathrm{AD}$ and the consequent scientific and social impact, current spatial memory research did not focus on other MCI types and etiologies. Recent researches showed that egocentric and allocentric memory can be impaired in other neurodegenerative diseases, such as vascular [61, 62], Lewy bodies [58], frontotemporal [59], and even Parkinson's disease [60, 111]. Additionally, subjective navigation complaints have been reported in individuals with naMCI and subjective cognitive decline [63]. Hence, future research should strengthen/expand aMCI findings but also focus on MCI with other underlining diseases (e.g., [112-115]). Defining the underlining etiology is crucial for differential diagnosis among MCI subtypes, for instance striatal lesions detected with Fazekas's 
criteria impacted egocentric memory of both $\mathrm{HC}$ and aMCI. This is consistent with initial findings of spatial memory deficits in vascular cognitive impairment [61, 62]; moreover, vascular risk factors for AD were also found to affect egocentric memory as well [107].

Learning and recalling spatial information is affected in MCI and the severity of these deficits can be located between the performance of $\mathrm{HC} /$ subjective cognitive decline and AD individuals. This is consistent with the notion of MCI as a transitional stage between aging and dementia [51], where also spatial memory and navigation should be considered as affected domains of this subclinical entity [116]. Impairment in allocentric memory is in line with previous neuropathological and neuropsychological alterations in MCI and $\mathrm{AD}[40,116]$ Consistently, the studies included that investigated neural underpinnings showed hippocampal (particularly on the right side), and more general medial temporal, involvement. This is consistent with the consolidated role of these regions in spatial memory [117-119]. Interestingly, we found also egocentric deficits that could be related in aMCI to reduced activation of the caudate and the precuneus during spatial navigation task [120]. Indeed, these regions are crucial for egocentric navigation $[22,121]$ and spatial visual imagery (the so called "parietal window") [13]. The egocentric deficit could be exacerbated by neuropsychiatric comorbidities in aMCI due to fronto-striatal alterations [104, 122]. Only one study [55] compared egocentric versus allocentric memory and, surprisingly, found that egocentric memory was worst in $\mathrm{AD}$, aMCI, and HC compared to allocentric memory. This is in contrast with egocentric navigation preference in aging and MCI $[33,76]$. However, to successfully navigate the environment a flexible combination and switching of the two navigation modes is required [29, 33, 116]. Importantly, only one study assess allocentric dependent-independent view-point abilities after navigation [54], a critical point as switching abilities between spatial frames is crucial for navigation and is impaired in AD individuals [24, 40, 116, 123]. However, aMCI preferred learning was affected only for allocentric memory, indeed aMCI due to AD pathology preferred egocentric rather than allocentric strategy.

More specifically, we found that aMCImd has greater impairment of both types of spatial memory (learning, immediate, and delayed recall), similar to $\mathrm{AD}$, compared to aMCIsd but the latter should be considered as a hippocampal subtype. Indeed, spatial navigation requires a large network of brain regions and cognitive functions to successfully navigate and recall the environment (for a review, see [28]), hence aMCImd could have poorer performance as multiple neuropsychological functions are impaired. Conversely, by a neuroanatomical point of view, aMCIsd could be conceptualized as a 'pure' allocentric phenotype [96]. This resembles the pattern of $\mathrm{H}$ and $\mathrm{NH}$ aMCI, distinguished by means of the FCSRT [41]. We found that HaMCI had similar performances to $\mathrm{AD}$ on spatial learning and retrieval, whereas NHaMCI had less pronounced deficits in navigation. HaMCI and aMCIsd could be thought as prodromal 'hippocampal' stages of AD $[41,116]$ and thus it would be possible to improve diagnosis, prognosis and treatment. Indeed, AD pathology affects not only memory per se but the neural substrate of idiothetic navigation itself and spatial behavior as noted by differences in A + and A-individuals [78]. This finding is also interesting within the context of embodiment in spatial memory, where multisensory cues play a critical role for representation of the body and the space $[2,86,90]$. Indeed, embodiment might lead to alterations in egocentric and allocentric abilities in normal and pathological (i.e., AD and Parkinson's disease) aging [31, 68, 124] and spatial memory can be improved by adopting active navigation as shown by a recent systematic review of Tuena and colleagues [74].

Given the crucial role in pathological aging, spatial memory could be used as a marker of AD pathology to improve differential diagnosis. Individuals with genetic markers of $\mathrm{AD}$, such as $\varepsilon 4$ and $\varepsilon 3$ (VL) carriers, have particular deficits in recalling spatial memory rather than in spatial learning [53, 71, 93]. Allocentric and delayed tests impairment are associated with VL compared to S poly-T homopolymer, whereas $\varepsilon 4$ carriers can be distinguished from noncarriers in egocentric and allocentric memory. More, egocentric memory can tap subtle differences among heterozygous, homozygous and non-carriers. Preserved egocentric abilities can also be a marker of individual with aMCI but without A pathology. However, both types of spatial memory can successfully discriminate aMCI individuals with AD pathology from those who have only neuropsychological marker of aMCI [78].

Finally, delayed recall of allocentric memory is crucial for distinguishing aMCI of sd, $\mathrm{md} \mathrm{H}$ and $\mathrm{NH}$ types from $\mathrm{HC}$ and $\mathrm{AD}$, between $\mathrm{H}$ and $\mathrm{NH}$ amnesias and S-VL/VL and S/S carriers but not between $\varepsilon 4$ carriers and non-carriers. Conversely, mixed performances show also this patter for $\varepsilon 4$ carriers and 
non-carriers but not between aMCIsd and HC. It is interesting to note that in this subtask the individual is provided with both types of spatial frames and consequently could use the preferred navigation strategy. Consequently, by forcing the adoption of one of the spatial frames the results become consistent between the groups and tap specific impairments like the 'pure' allocentric deficit in aMCIsd.

Unfortunately, as some studies did not provide full egocentric versus allocentric performances, MCI subtypes comparisons or did not stratify the MCI population, future research should provide at least neuropsychological profiles and their statistical differences or trends of their spatial memory.

The most used navigation task real-world or virtual version of the human analogue (HGT) of the Morris water maze [70]. However, other studies used other spatial navigation tasks such as the frame matching task by Serino and colleagues [54], the large-scale real-world environment in Schöberl and co-authors [78] or the active versus passive navigation semiimmersive scenario in Plancher and collaborators [106]. Involvement of different research groups and paradigms would reduce bias and improve basic and clinical research in this topic. On the one hand, it is crucial for replicability to use consolidated tasks, on the other hand future research would benefit from more heterogenous navigational tasks. For instance, by means of discrete landmark/egocentric and boundary landmark/allocentric navigation strategies [22, $29,66,119]$, it is possible to tap procedural (striatal) and declarative (hippocampal) spatial memory; this paradigm could be applied to study diseases where one or both the neuroanatomical underpinnings are affected by certain diseases (e.g., Parkinson, Lewy body, WMH).

Surprisingly, our results show that real and virtual versions can provide similar results across aMCI subtypes. Vision is the most salient sensory input provided for navigation, and visual cues are indeed the most critical reference when self-motion cannot provide correct spatial orientation [11, 90, 125]. However, some subtasks might involve other memory systems than immediate and delayed memory, such as short-term visual memory. This is consistent with findings showing that path integration and spatial memory involve medial temporal regions only when adequate number of items and delay are used for encoding and retrieval $[80,126]$. Nevertheless, we think that current VR solutions still lack high degrees of virtual embodiment by means of semi-immersive/immersive apparatus. By involv- ing embodiment and multisensory inputs [86, 87] it could be possible to improve idiothetic features (e.g., proprioception, interoception, vestibular information) for simulating real-life navigation [89, 127].

Portable neuroimaging/neuromodulation devices could be used to study real time navigation and spatial memory $[128,129]$; indeed, studies reported here use 'off-line' paradigms. However, our results are in line with previous research concerning the neuropsychology of spatial memory $[10,13,118]$ and $\mathrm{AD}$ models [39, 40, 116, 123]. Interestingly, Schöberl and colleagues [78] showed that 'active navigation' has different metabolic pattern than locomotion and that navigation regions are impaired in MCI. Using regional cerebral glucose metabolism (rCGM), they compared navigation with locomotion (locomotion only without navigation task) and found that involved different brain regions (right anterior hippocampus, bilateral RSC, and pontine tegmentum) in healthy participants. In aMCI patients a relative increase of rCGM in the pontine tegmentum and in the right anterior hippocampus in the control group was found. $\mathrm{A}+\mathrm{aMCI}$ showed lower rCGM during navigation compared to locomotion in the right anterior hippocampus, bilateral RSC cortex, precuneus, and parietal lobe compared to controls. A + compared to A- aMCI group revealed reduced rCGM in the bilateral hippocampus and right superior parietal lobule and increased metabolism in the pontomedullary tegmentum and left frontal lobe. Again, Schöberl and colleagues [78] found that both A + and A-groups had lower gait speed during navigation compared to controls. The findings are consistent with the critical role of active navigation components used to derive spatial information [4] and that the use of both bodily and cognitive factors during exploration could be included in memory assessment and rehabilitation for pathological embodiment in aging [1, 31, 74].

Again, Schöberl and colleagues [78] explored visual exploration in aMCI individuals finding that A- patients had more lateral and total fixations and total, egocentric, allocentric, and search saccades than A + individuals. A- group fixed fewer unique landmarks during allocentric task than controls and $\mathrm{A}+$ patients, and $\mathrm{A}+$ and $\mathrm{A}-$ spent more time at crossings and neglected shortcuts compared to control group. Concerning memory tests, the authors showed that overall, egocentric and allocentric navigation performances differentiate $\mathrm{A}+$ from $\mathrm{A}-$, rather than auditory-verbal learning, executive functions, and global cognition tests. In particular, the FCSRT has an important role when combined with spatial 
memory measures for diagnosis of MCI [41, 94, 108]. Further studies should deepen the diagnostic, and consequently cognitive rehabilitation, peculiarities of MCI profiles, as spatial memory combined with neuropsychological tests and behavioral information might help to better differentiate this complex entity.

Behavioral measures are very useful for studying MCI and dementia. Indeed, if on the one hand neuropsychological tests of memory and visuospatial functions were associated with allocentric and egocentric memory respectively and spatial rotation with frames switching abilities [54, 107, 110], on the other hand, egocentric and allocentric memory should be investigated and treated as separate entities ("Spatial Navigation"), which has important consequences for diagnostic and rehabilitative purposes. Indeed, Laczò and colleagues [110] conducted an exploratory factor analysis that revealed that allocentric and egocentric navigation scores loaded on their own factor, separately from the other cognitive functions (verbal and non-verbal memory, executive and visuospatial function, attention/working memory and language function).

Limitations of current spatial memory research in MCI could be summarized in the need to: 1) consider different MCI etiologies and/or neuropsychological deficits in assessing spatial memory and expand researches on aMCI/MCI due to AD with larger sample size; 2) employ other tasks besides HGT, including also the assessment of switching abilities; 3) improve the VR tasks, including idiothetic cues, full-immersive solutions with comparison with real versions; 4) assess all the differences and trends within the MCI typologies (naMCI included), frame switching abilities and the potential deficits in embodiment (virtual embodiment when using VR) in this sample and how they affect spatial memory and active navigation; and 5) improve blinding of outcome measures, state any missing data and explain how it was handled, add power analysis, improve the control over confounding variables and clarify in detail MCI subtypes/spatial memory differences in hypotheses and results.

\section{Conclusions}

Although egocentric and allocentric spatial memory decline has been deeply investigated in both healthy older people [32] and in AD individuals [40], to our knowledge a systematic review focusing on MCI patients is lacking. aMCI is the most studied subtype give its importance for $\mathrm{AD}$ and spatial memory with deficits in both egocentric and allocentric memory; aMCI with AD markers shows specific spatial memory impairments instead. However, future research could investigate how navigation could be impaired in other MCI subtypes. This can be done by using VR and real-world tasks. In particular, VR solutions showed good usability in older people and it could be useful to investigate how bodily information (embodiment) relates to spatial cognition, also combined with artificial intelligence methods, to improve diagnosis and treatment [130-142]. In conclusion, identifying cognitive markers typical of this transitional condition might allow also early neurorehabilitation interventions to prevent the progression to dementia.

\section{ACKNOWLEDGMENTS}

This manuscript is supported by the Italian Ministry of Health (project code: SG-2018-12368175).

Authors' disclosures available online (https:// www.j-alz.com/manuscript-disclosures/20-1017r1).

\section{SUPPLEMENTARY MATERIAL}

The supplementary material is available in the electronic version of this article: https://dx.doi.org/ 10.3233/JAD-201017.

\section{REFERENCES}

[1] Lester AW, Moffat SD, Wiener JM, Barnes CA, Wolbers T (2017) The aging navigational system. Neuron 95, 10191035.

[2] Tcheang L, Bülthoff HH, Burgess N (2011) Visual influence on path integration in darkness indicates a multimodal representation of large-scale space. Proc Natl Acad Sci U $S$ A 108, 1152-1157.

[3] Barry C, Burgess N (2014) Neural mechanisms of selflocation. Curr Biol 24, R330-R339.

[4] Chrastil ER, Warren WH (2012) Active and passive contributions to spatial learning. Psychon Bull Rev 19, 1-23.

[5] Wilson PN, Foreman N, Gillett R, Stanton D (1997) Active versus passive processing of spatial information in a computer-simulated environment. Ecol Psychol 9, 37-41.

[6] Keefe JO, Nadel L (1978) The Hippocampus as a Cognitive Map, Clarendon Press, Oxford.

[7] Tolman EC (1948) Cognitive maps in rats and men. Psychol Rev 55, 189-208.

[8] American Psychological Association (2007) APA Dictionary of Psychology.

[9] Klatzky RL (1998) Allocentric and egocentric spatial representations: Definitions, distinctions, and interconnections. In Spatial cognition, Springer Berlin Heidelberg, pp. 1-17. 
[10] Burgess N (2008) Spatial cognition and the brain. Ann N Y Acad Sci 97, 77-97.

[11] Poulter S, Hartley T, Lever C (2018) The neurobiology of mammalian navigation. Curr Biol 28, R1023-R1042.

[12] Hartley T, Lever C, Burgess N, O'Keefe J (2014) Space in the brain: How the hippocampal formation supports spatial cognition. Philos Trans R Soc Lond B Biol Sci 369, 20120510.

[13] Byrne P, Becker S, Burgess N (2009) Remembering the past and imagining the future: A neural model of spatial memory and imagery. Psychol Rev 114, 340-375.

[14] O'Keefe J, Dostrovsky J (1971) The hippocampus as a spatial map. Preliminary evidence from unit activity in the freely-moving rat. Brain Res 34, 171-175.

[15] Ekstrom AD, Kahana MJ, Caplan JB, Fields TA, Isham EA, Newman EL, Fried I (2003) Cellular networks underlying human spatial navigation. Nature 425, 184-188.

[16] Jacobs J (2014) Hippocampal theta oscillations are slower in humans than in rodents: Implications for models of spatial navigation and memory. Philos Trans $R$ Soc Lond $B$ Biol Sci 369, 20130304.

[17] Hafting T, Fyhn M, Molden S, Moser MB, Moser EI (2005) Microstructure of a spatial map in the entorhinal cortex. Nature 436, 801-806.

[18] Cullen KE, Taube JS (2017) Our sense of direction: Progress, controversies and challenges. Nat Neurosci 20, 1466-1473.

[19] Lever C, Burton S, Jeewajee A, O'Keefe J, Burgess N (2009) Boundary vector cells in the subiculum of the hippocampal formation. J Neurosci 29, 9771-9777.

[20] Wolbers T, Bu C (2005) Dissociable retrosplenial and hippocampal contributions to successful formation of survey representations. J Neurosci 25, 3333-3340.

[21] Zhang H, Ekstrom A (2013) Human neural systems underlying rigid and flexible forms of allocentric spatial representation. Hum Brain Mapp 34, 1070-1087.

[22] Chersi F, Burgess N (2015) The cognitive architecture of spatial navigation: Hippocampal and striatal contributions. Neuron 88, 64-77.

[23] Mizumori SJY, Puryear CB, Martig AK (2009) Basal ganglia contributions to adaptive navigation. Behav Brain Res 199, 32-42.

[24] Vann SD, Aggleton JP, Maguire EA (2009) What does the retrosplenial cortex do? Nat Rev Neurosci 10, 792-802.

[25] Wolbers T, Hegarty M (2010) What determines our navigational abilities? Trends Cogn Sci 14, 138-146.

[26] Zaehle T, Jordan K, Wüstenberg T, Baudewig J, Dechent P, Mast FW (2007) The neural basis of the egocentric and allocentric spatial frame of reference. Brain Res $\mathbf{1 1 3 7}$, 92-103.

[27] Buckner RL, Carroll DC (2007) Self-projection and the brain. Trends Cogn Sci 11, 49-57.

[28] Cona G, Scarpazza C (2019) Where is the "where" in the brain? A meta-analysis of neuroimaging studies on spatial cognition. Hum Brain Mapp 40, 1867-1886.

[29] Doeller CF, King JA, Burgess N (2008) Parallel striatal and hippocampal systems for landmarks and boundaries in spatial memory. Proc Natl Acad Sci U S A 105, 59155920 .

[30] Techentin C, Voyer D, Voyer SD (2014) Spatial abilities and aging: A meta-analysis. Exp Aging Res 40, 395-425.

[31] Kuehn E, Perez-Lopez MB, Diersch N, Döhler J, Wolbers T, Riemer M (2017) Embodiment in the aging mind. Neurosci Biobehav Rev 86, 207-225.
[32] Colombo D, Serino S, Tuena C, Pedroli E, Dakanalis A, Cipresso P, Riva G (2017) Egocentric and allocentric spatial reference frames in aging: A systematic review. Neurosci Biobehav Rev 80, 605-621.

[33] Rodgers MK, Sindone JA 3rd, Moffat SD (2012) Effects of age on navigation strategy. Neurobiol Aging 33, 202.e15202.e2.

[34] Stangl M, Achtzehn J, Huber K, Dietrich C, Tempelmann C, Wolbers T (2018) Compromised grid-cell-like representations in old age as a key mechanism to explain age-related navigational deficits. Curr Biol 28, 11081115.e6.

[35] Lithfous S, Dufour A, Després O (2013) Spatial navigation in normal aging and the prodromal stage of Alzheimer's disease: Insights from imaging and behavioral studies. Ageing Res Rev 12, 201-213.

[36] Braak H, Braak E (1991) Neuropathological stageing of Alzheimer-related changes. Acta Neuropathol 82, 239-259.

[37] Jagust W (2018) Imaging the evolution and pathophysiology of Alzheimer disease. Nat Rev Neurosci 19, 687-700.

[38] Jack CR, Bennett DA, Blennow K, Carrillo MC, Dunn B, Haeberlein SB, Holtzman DM, Jagust W, Jessen F, Karlawish J, Liu E, Molinuevo JL, Montine T, Phelps C, Rankin KP, Rowe CC, Scheltens P, Siemers E, Snyder HM, Sperling R, Elliott C, Masliah E, Ryan L, Silverberg N (2018) NIA-AA Research Framework: Toward a biological definition of Alzheimer's disease. Alzheimers Dement 14, 535-562.

[39] Vlček K, Laczó J (2014) Neural correlates of spatial navigation changes in mild cognitive impairment and Alzheimer's disease. Front Behav Neurosci 8, 6 .

[40] Serino S, Cipresso P, Morganti F, Riva G (2014) The role of egocentric and allocentric abilities in Alzheimer's disease: A systematic review. Ageing Res Rev 16, 32-44.

[41] Dubois B, Albert ML (2004) Amnestic MCI or prodromal Alzheimer's disease? Lancet Neurol 3, 246-248.

[42] Albert MS, Dekosky ST, Dickson D, Dubois B, Feldman HH, Fox NC, Gamst A, Holtzman DM, Jagust WJ, Petersen RC, Snyder PJ, Carrillo MC, Thies B, Phelps CH (2011) The diagnosis of mild cognitive impairment due to Alzheimer's disease: Recommendations from the National Institute on Aging-Alzheimer's Association workgroups on diagnostic guidelines for Alzheimer's disease. Alzheimers Dement 7, 270-279.

[43] Petersen RC, Smith GE, Ivnik RJ, Tangalos EG, Schaid DJ, Thibodeau SN, Kokmen E, Waring SC, Kurland LT (1995) Apolipoprotein E status as a predictor of the development of Alzheimer's disease in memory-impaired individuals. JAMA 273, 1274-1278.

[44] Petersen RC, Smith GE, Waring SC, Ivnik RJ, Tangalos EG, Kokmen E (1999) Mild cognitive impairment clinical characterization and outcome. Arch Neurol 56, 303-309.

[45] Petersen RC (2004) Mild cognitive impairment as a diagnostic entity. J Intern Med 256, 183-194.

[46] Graham JE, Rockwood K, Beattie BL, Eastwood R, Gauthier S, Tuokko H, Mcdowell I (1997) Prevalence and severity of cognitive impairment with and without dementia in an elderly population. Lancet 349, 1793-1796.

[47] Dubois B, Feldman HH, Jacova C, Hampel H, Molinuevo JL, Blennow K, Dekosky ST, Gauthier S, Selkoe D, Bateman R, Cappa S, Crutch S, Engelborghs S, Frisoni GB, Fox NC, Galasko D, Habert MO, Jicha GA, Nordberg A, Pasquier F, Rabinovici G, Robert P, Rowe C, Salloway S, 
Sarazin M, Epelbaum S, de Souza LC, Vellas B, Visser PJ, Schneider L, Stern Y, Scheltens P, Cummings JL (2014) Advancing research diagnostic criteria for Alzheimer's disease: The IWG-2 criteria. Lancet Neurol 13, 614629.

[48] Hixson JE, Vernier DT (1990) Restriction isotyping of human apolipoprotein $\mathrm{E}$ by gene amplification and cleavage with HhaI. $J$ Lipid Res 31, 545-548.

[49] Roses AD, Lutz MW, Amrine-Madsen H, Saunders AM, Crenshaw DG, Sundseth SS, Huentelman MJ, Welsh-Bohmer KA, Reiman EM (2010) A TOMM40 variable-length polymorphism predicts the age of lateonset Alzheimer's disease. Pharmacogenomics $J \mathbf{1 0}$, 375-384.

[50] Fazekas F, Chawluk JB, Alavi A, Hurtig HI, Zimmerman RA (1987) MR signal abnormalities at $1.5 \mathrm{~T}$ in Alzheimer's dementia and normal aging. Am J Radiol 149, 351-356.

[51] Vega JN, Newhouse PA (2014) Mild cognitive impairment: Diagnosis, longitudinal course, and emerging treatments. Curr Psychiatry Rep 16, 490-498.

[52] Petersen RC (2016) Mild cognitive impairment. Continuum (Minneap Minn) 22 (2 Dementia), 404-418.

[53] Laczó J, Andel R, Vyhnalek M, Matoska V, Kaplan V, Nedelska Z, Lerch O, Gazova I, Moffat SD, Hort J (2015) The effect of TOMM40 on spatial navigation in amnestic mild cognitive impairment. Neurobiol Aging 36, 20242033.

[54] Serino S, Morganti F, Di Stefano F, Riva G (2015) Detecting early egocentric and allocentric impairments deficits in Alzheimer's disease: An experimental study with virtual reality. Front Aging Neurosci 7, 88.

[55] Mohammadi A, Kargar M, Hesami E (2018) Using virtual reality to distinguish subjects with multiple- but not singledomain amnestic mild cognitive impairment from normal elderly subjects. Psychogeriatrics 18, 132-142.

[56] De Santi S, De Leon MJ, Rusinek H, Convit A, Tarshish CY, Roche A, Tsui WH, Kandil E, Boppana M, Daisley K, Wang GJ, Schlyer D, Fowler J (2001) Hippocampal formation glucose metabolism and volume losses in MCI and AD. Neurobiol Aging 22, 529-539.

[57] Pengas G, Hodges JR, Watson P, Nestor PJ (2010) Focal posterior cingulate atrophy in incipient Alzheimer's disease. Neurobiol Aging 31, 25-33.

[58] Nedelska Z, Laczo J, Uller M, Vyhnalek M, Fripp J, Vlcek K, Parizkova M, Hort J (2017) Dementia with lewy bodies: Severe impairment of real-space navigation skills examined with human analogue of morris water maze and their structural underpinnings. J Neurol Sci 381, 83-84.

[59] Tu S, Spiers HJ, Hodges JR, Piguet O, Hornberger M (2017) Egocentric versus allocentric spatial memory in behavioral variant frontotemporal dementia and Alzheimer's disease. J Alzheimers Dis 59, 883-892.

[60] Thurm F, Schuck NW, Fauser M, Doeller CF, Stankevich Y, Evens R, Riedel O, Storch A, Lueken U, Li SC (2016) Dopamine modulation of spatial navigation memory in Parkinson's disease. Neurobiol Aging 38, 93-103.

[61] Coughlan G, Flanagan E, Jeffs S, Bertoux M, Spiers H, Mioshi E, Hornberger M (2018) Diagnostic relevance of spatial orientation for vascular dementia. A case study. Dement Neuropsychol 12, 85-91.

[62] Lowry E, Puthusseryppady V, Coughlan G, Jeffs S, Hornberger M (2020) Path integration changes as a cognitive marker for vascular cognitive impairment? - A pilot study. Front Hum Neurosci 14, 131.

[63] Cerman J, Andel R, Laczo J, Vyhnalek M, Nedelska Z, Mokrisova I, Sheardova K, Hort J (2018) Subjective spatial navigation complaints - a frequent symptom reported by patients with subjective cognitive decline, mild cognitive impairment and Alzheimer's disease. Curr Alzheimer Res 15, 219-228.

[64] Bosco A, Picucci L, Caffò AO, Lancioni GE, Gyselinck V (2008) Assessing human reorientation ability inside virtual reality environments: The effects of retention interval and landmark characteristics. Cogn Process 9, 299-309.

[65] Bohil CJ, Alicea B, Biocca FA (2011) Virtual reality in neuroscience research and therapy. Nat Rev Neurosci 12, $752-762$.

[66] Guderian S, Dzieciol XAM, Gadian DG, Jentschke XS, Doeller CF, Burgess N, Mishkin XM, Vargha-khadem F (2015) Hippocampal volume reduction in humans predicts impaired allocentric spatial memory in virtual-reality navigation. J Neurosci 35, 14123-14131.

[67] Serino S, Riva G (2013) Getting lost in Alzheimer's disease: A break in the mental frame syncing. Med Hypotheses 80, 416-421.

[68] Serino S, Riva G (2016) The proactive self in space: How egocentric and allocentric spatial impairments contribute to anosognosia in Alzheimer's disease. J Alzheimers Dis 55, 881-892.

[69] Riva G, Di Lernia D, Serino A, Serino S (2019) The role of reference frames in memory recollection. Behav Brain Sci 42, e296.

[70] Morris RGM, Garrud P, Rawlins JNP, O'Keefe J (1982) Place navigation impaired in rats with hippocampal lesions. Nature 297, 681-683.

[71] Laczó J, Andel R, Vyhnalek M, Vlcek K, Nedelska Z, Matoska V, Gazova I, Mokrisova I, Sheardova K, Hort J (2014) APOE and spatial navigation in amnestic MCI: Results from a computer-based test. Neuropsychology 28, 676-684.

[72] Weniger G, Ruhleder M, Lange C, Wolf S, Irle E (2011) Egocentric and allocentric memory as assessed by virtual reality in individuals with amnestic mild cognitive impairment. Neuropsychologia 49, 518-527.

[73] Howett D, Castegnaro A, Krzywicka K, Hagman J, Marchment D, Henson R, Rio M, King JA, Burgess N, Chan D (2019) Differentiation of mild cognitive impairment using an entorhinal cortex-based test of virtual reality navigation. Brain 142, 1751-1766.

[74] Tuena C, Serino S, Dutriaux L, Riva G, Piolino P (2019) Virtual enactment effect on memory in young and aged populations: A systematic review. J Clin Med 8, 620.

[75] Laczó J, Andel R, Vyhnalek M, Vlcek K, Magerova H, Varjassyova A, Nedelska Z, Gazova I, Bojar M, Sheardova K, Hort J (2012) From Morris water maze to computer tests in the prediction of Alzheimer's disease. Neurodegener Dis 10, 153-157.

[76] Parizkova M, Lerch O, Moffat SD, Andel R, Mazancova AF, Nedelska Z, Vyhnalek M, Hort J, Laczó J (2018) The effect of Alzheimer's disease on spatial navigation strategies. Neurobiol Aging 64, 107-115.

[77] King EG (2011) Age differences and spatial navigation in novel virtual and real world environments (Thesis). University of Florida, http://ufdc.ufl.edu/UFE0041348/00001

[78] Schöberl F, Pradhan C, Irving S, Buerger K, Xiong G, Kugler G, Kohlbecher S, Engmann J, Werner P, 
Brendel M, Schneider E, Perneczky R, Jahn K, la Fougère $C$, Bartenstein $P$, Brandt $T$, Dieterich $M$, Zwergal A (2020) Real-space navigation testing differentiates between amyloid-positive and -negative aMCI. Neurology 94, e861-e873.

[79] DeIpolyi AR, Rankin KP, Mucke L, Miller BL, GornoTempini ML (2007) Spatial cognition and the human navigation network in AD and MCI. Neurology 69, 986-997.

[80] Shrager Y, Kirwan CB, Squire LR (2008) Neural basis of the cognitive map: Path integration does not require hippocampus or entorhinal cortex. Proc Natl Acad Sci U S A 105, 12034-12038.

[81] Repetto C, Serino S, Macedonia M, Riva G (2016) Virtual reality as an embodied tool to enhance episodic memory in elderly. Front Psychol 7, 1839.

[82] Slater M, Wilbur S (1997) A framework for immersive virtual environments (FIVE): Speculations on the role of presence in virtual environments. Presence Teleoperators Virtual Environ 6, 603-616.

[83] Riva G, Waterworth JA (2014) Being present in a virtual world. In The Oxford Handbook of Virtuality, Grimshaw M, ed. Oxford University Press.

[84] Jebara N, Orriols E, Zaoui M, Berthoz A, Piolino P (2014) Effects of enactment in episodic memory: A pilot virtual reality study with young and elderly adults. Front Aging Neurosci 6, 338 .

[85] Riva G (2008) From virtual to real body: Virtual reality as embodied technology. J Cyber Ther Rehabil 1, 7-22.

[86] Riva G (2018) The neuroscience of body memory: From the self through the space to the others. Cortex 104, 241-260.

[87] Riva G, Wiederhold BK, Mantovani F (2019) Neuroscience of virtual reality: From virtual exposure to embodied medicine. Cyberpsychol Behav Soc Netw 22, 82-96.

[88] Kilteni K, Groten R, Slater M (2012) The sense of embodiment in virtual reality. Presence Teleoperators Virtual Environ 21, 373-387.

[89] Taube JS, Valerio S, Yoder RM (2013) Is navigation in virtual reality with fMRI really navigation? J Cogn Neurosci 25, 1008-1019.

[90] Chen G, King JA, Burgess N, O'Keefe J (2013) How vision and movement combine in the hippocampal place code. Proc Natl Acad Sci U S A 110, 378-383.

[91] Ramanoël S, York E, Le Petit M, Lagrené K, Habas C, Arleo A (2019) Age-related differences in functional and structural connectivity in the spatial navigation brain network. Front Neural Circuits 13, 69.

[92] Moher D, Liberati A, Tetzlaff J, Altman D (2009) Preferred reporting items for systematic reviews and meta-analyses: The PRISMA statement. Ann Intern Med 151, 264269.

[93] Laczó J, Andel R, Vlček K, Macoška V, Vyhnálek M, Tolar M, Bojar M, Hort J (2011) Spatial navigation and APOE in amnestic mild cognitive impairment. Neurodegener Dis 8, 169-177.

[94] Laczó J, Andel R, Vyhnalek M, Vlcek K, Magerova H, Varjassyova A, Tolar M, Hort J (2010) Human analogue of the Morris water maze for testing subjects at risk of Alzheimer's disease. Neurodegener Dis 7, 148-152.

[95] Methley AM, Campbell S, Chew-Graham C, McNally R, Cheraghi-Sohi S (2014) PICO, PICOS and SPIDER: A comparison study of specificity and sensitivity in three search tools for qualitative systematic reviews. $B M C$ Health Serv Res 14, 579.

[96] Hort J, Laczó J, Vyhnálek M, Bojar M, Bureš J, Vlček K (2007) Spatial navigation deficit in amnestic mild cognitive impairment. Proc Natl Acad Sci U S A 104, 4042-4047.

[97] Higgins JP, Altman DG, Gøtzsche PC, Jüni P, Moher D, Oxman AD, Savovic J, Schulz KF, Weeks L, Sterne JA (2011) The Cochrane Collaboration's tool for assessing risk of bias in randomised trials. BMJ 343, d5928.

[98] Rockers PC, Røttingen JA, Shemilt I, Tugwell P, Bärnighausen $T$ (2015) Inclusion of quasi-experimental studies in systematic reviews of health systems research. Health Policy 119, 511-521.

[99] Downs SH, Black N (1998) The feasibility of creating a checklist for the assessment of the methodological quality both of randomised and non-randomised studies of health care interventions. J Epidemiol Community Health 52, 377-384.

[100] Hooper P, Jutai JW, Strong G, Russell-Minda E (2008) Age-related macular degeneration and low-vision rehabilitation: A systematic review. Can J Ophthalmol 43, 180-187.

[101] Petersen RC, Doody R, Kurz A, Mohs RC, Morris JC, Rabins P V., Ritcihie K, Rossor M, Thal L, Winblad B (2001) Current concepts in mild cognitive impairment. Arch Neurol 58, 1985-1992.

[102] Petersen RC, Negash S (2008) Mild cognitive impairment: An overview. CNS Spectr 13, 45-53.

[103] WHO (1992) The ICD-10 classification of mental and behavioural disorders: Clinical descriptions and diagnostic guidelines. Geneva.

[104] Keynejad RC, Marková H, Šiffelová K, Kumar N, Vlček K, Laczó J, Migo EM, Hort J, Kopelman MD (2018) Spatial navigation deficits in amnestic mild cognitive impairment with neuropsychiatric comorbidity. Aging Neuropsychol Cogn 25, 277-289.

[105] Yesavage JA (1988) Geriatric depression scale. Psychopharmacol Bull 24, 709-711.

[106] Plancher G, Tirard A, Gyselinck V, Nicolas S, Piolino P (2012) Using virtual reality to characterize episodic memory profiles in amnestic mild cognitive impairment and Alzheimer's disease: Influence of active and passive encoding. Neuropsychologia 50, 592-602.

[107] Parizkova M, Andel R, Lerch O, Marková H, Gažová I, Vyhnálek M, Hort J, Laczó J (2017) Homocysteine and real-space navigation performance among non-demented older adults. J Alzheimers Dis 55, 951-964.

[108] Laczó J, Vlček K, Vyhnálek M, Vajnerová O, Ort M, Holmerová I, Tolar M, Andel R, Bojar M, Hort J (2009) Spatial navigation testing discriminates two types of amnestic mild cognitive impairment. Behav Brain Res 202, 252-259.

[109] Qing Z, Li W, Nedelska Z, Wu W, Wang F, Liu R, Zhao H, Chen W, Chan Q, Zhu B, Xu Y, Hort J, Zhang B (2017) Spatial navigation impairment is associated with alterations in subcortical intrinsic activity in mild cognitive impairment: A resting-state fMRI study. Behav Neurol 2017, 6364314.

[110] Laczó J, Andel R, Nedelska Z, Vyhnalek M, Vlcek K, Crutch S, Harrison J, Hort J (2017) Exploring the contribution of spatial navigation to cognitive functioning in older adults. Neurobiol Aging 51, 67-70.

[111] Schneider CB, Linse K, Schönfeld R, Brown S, Koch R, Reichmann H, Leplow B, Storch A (2017) Spatial learning 
deficits in Parkinson's disease with and without mild cognitive impairment. Parkinsonism Relat Disord 36, 83-88.

[112] De Mendonça A, Ribeiroa F, Guerreiro M, Garcia C (2004) Frontotemporal mild cognitive impairment. J Alzheimers Dis 6, 1-9.

[113] Litvan I, Goldman JG, Tröster AI, Schmand BA, Weintraub D, Petersen RC, Mollenhauer B, Adler CH, Marder K, Williams-Gray CH, Aarsland D, Kulisevsky J, Rodriguez-Oroz MC, Burn DJ, Barker RA, Emre M (2012) Diagnostic criteria for mild cognitive impairment in Parkinson's disease: Movement Disorder Society Task Force guidelines. Mov Disord 27, 349-356.

[114] McKeith IG, Ferman TJ, Thomas AJ, Blanc F, Boeve BF, Fujishiro H, Kantarci K, Muscio C, O’Brien JT, Postuma RB, Aarsland D, Ballard C, Bonanni L, Donaghy P, Emre M, Galvin JE, Galasko D, Goldman JG, Gomperts SN, Honig LS, Ikeda M, Leverenz JB, Lewis SJG, Marder KS, Masellis M, Salmon DP, Taylor JP, Tsuang DW, Walker Z, Tiraboschi P (2020) Research criteria for the diagnosis of prodromal dementia with Lewy bodies. Neurology 94, 743-755.

[115] Skrobot OA, Black SE, Chen C, DeCarli C, Erkinjuntti T, Ford GA, Kalaria RN, O’Brien J, Pantoni L, Pasquier F, Roman GC, Wallin A, Sachdev P, Skoog I; VICCCS group, Ben-Shlomo Y, Passmore AP, Love S, Kehoe PG (2018) Progress toward standardized diagnosis of vascular cognitive impairment: Guidelines from the Vascular Impairment of Cognition Classification Consensus Study. Alzheimers Dement 14, 280-292.

[116] Coughlan G, Laczó J, Hort J, Minihane A-M, Hornberger M (2018) Spatial navigation deficits - overlooked cognitive marker for preclinical Alzheimer disease? Nat Rev Neurol 14, 496-506.

[117] Nedelska Z, Andel R, Laczó J, Vlcek K, Horinek D, Lisy J, Sheardova K, Bureš J, Hort J (2012) Spatial navigation impairment is proportional to right hippocampal volume. Proc Natl Acad Sci U S A 109, 2590-2594.

[118] Burgess N, Maguire EA, O'Keefe J (2002) The human hippocampus and spatial and episodic memory. Neuron 35, 625-641.

[119] Iglói K, Doeller CF, Berthoz A, Rondi-Reig L, Burgess N (2010) Lateralized human hippocampal activity predicts navigation based on sequence or place memory. Proc Natl Acad Sci U S A 107, 14466-14471.

[120] Migo EM, O'Daly O, Mitterschiffthaler M, Antonova E, Dawson GR, Dourish CT, Craig KJ, Simmons A, Wilcock GK, McCulloch E, Jackson SH, Kopelman MD, Williams SC, Morris RG (2016) Investigating virtual reality navigation in amnestic mild cognitive impairment using fMRI. Neuropsychol Dev Cogn B Aging Neuropsychol Cogn 23, 196-217.

[121] Goodroe SC, Starnes J, Brown TI (2018) The complex nature of hippocampal-striatal interactions in spatial navigation. Front Hum Neurosci 12, 250.

[122] Bonelli RM, Cummings JL (2007) Frontal-subcortical circuitry and behavior. Dialogues Clin Neurosci 9, 141-151.

[123] Ruggiero G, Iachini T (2018) Editorial: Spatial cognition in normal aging, MCI and AD. Curr Alzheimer Res 15, 202-204.

[124] Arzy S, Schacter DL (2019) Self-agency and selfownership in cognitive mapping. Trends Cogn Sci 23, 476-487.

[125] Valerio S, Taube JS (2012) Path integration: How the head direction signal maintains and corrects spatial orientation. Nat Neurosci 15, 1445-1453.
[126] Shrager Y, Bayley PJ, Bontempi B, Hopkins RO, Squire LR (2007) Spatial memory and the human hippocampus. Proc Natl Acad Sci U S A 104, 2961-2966.

[127] Bohbot VD, Copara MS, Gotman J, Ekstrom AD (2017) Low-frequency theta oscillations in the human hippocampus during real-world and virtual navigation. Nat Commun 8, 14415 .

[128] Teo W-P, Muthalib M, Yamin S, Hendy AM, Bramstedt K, Kotsopoulos E, Perrey S, Hazan A (2016) Does a combination of virtual reality, neuromodulation and neuroimaging provide a comprehensive platform for neurorehabilitation? - a narrative review of the literature. Front Hum Neurosci 10, 284.

[129] Park JL, Dudchenko PA, Donaldson DI (2018) Navigation in real-world environments: New opportunities afforded by advances in mobile brain imaging. Front Hum Neurosci 12, 361 .

[130] Matamala-Gomez M, Malighetti C, Cipresso P, Pedroli E, Realdon O, Mantovani F, Riva G (2020) Changing body representation through full body ownership illusions might foster motor rehabilitation outcome in patients with stroke. Front Psychol 11, 1962.

[131] Chirico A, Malighetti C, Serino S, Cipresso P, Pedroli E, Tuena C, Muratore M, Riva G (2019) Towards an advancement of multisensory integration deficits in anorexia nervosa: Exploring temporal discrimination processing of visuo-auditory stimuli. Annu Rev CyberTherapy Telemed 17, 53-58.

[132] Malighetti C, Serino S, Riva G, Cipolletta S (2016) Inside and outside the self. Virtual reality and repertory grids in the spatial analysis of anorexic patients' meanings. Annu Rev CyberTherapy Telemed 14, 78-83.

[133] Winter D, Malighetti C, Cipolletta S, Ahmed S, Benson B, Röhricht F (2018) Construing and body dissatisfaction in chronic depression: A study of body psychotherapy. Psychiatry Res 270, 845-851.

[134] Cipolletta S, Malighetti C, Serino S, Riva G, Winter D (2017) Intrapersonal, interpersonal, and physical space in anorexia nervosa: A virtual reality and repertory grid investigation. Psychiatry Res 252, 87-93.

[135] Tuena C, Mancuso V, Benzi IMA, Cipresso P, Chirico A, Goulene KM, Riva G, Stramba-Badiale M, Pedroli E (2020) Executive functions are associated with fall risk but not balance in chronic cerebrovascular disease. J Clin $\operatorname{Med} \mathbf{9}, 3405$.

[136] Tuena C, Pedroli E, Trimarchi PD, Gallucci A, Chiappini M, Goulene K, Gaggioli A, Riva G, Lattanzio F, Giunco F, Stramba-Badiale M (2020) Usability issues of clinical and research applications of virtual reality in older people: A systematic review. Front Human Neurosci 14, 93.

[137] Tuena C, Semonella M, Fernández-Álvarez J, Colombo D, Cipresso P (2020) Predictive Precision Medicine: Towards the Computational Challenge. In P5 eHealth: An Agenda for the Health Technologies of the Future, Pravettoni G, Triberti S ed. Springer, Cham.

[138] Inghilleri P, Riva G, Riva E (2015) Introduction: Positive change in global world: Creative individuals and complex societies. In Enabling Positive Change Flow and Complexity in Daily Experience, Inghilleri P, Riva G, Riva E ed. Berlin: De Gruyter Open.

[139] Serino S, Scarpina F, Dakanalis D, Keizer A, Pedroli E, Castelnuovo G, Chirico A, Catallo V, di Lernia D, Riva G (2018) The role of age on multisensory bodily experience: An experimental study with a virtual reality full-body illusion. Cyberpsychol Behav Soc 21, 304-310. 
[140] Tuena C, Serino S, Gaston-Bellegarde A, Orriols E, Makowski D, Riva G, Piolino P (2017) How virtual embodiment affects episodic memory functioning: A proof-of-concept study. Annu Rev Cyberther Telemed 15, 98-103.

[141] Riva G, Wiederhold BK, Chirico A, Di Lernia D, Mantovani F, Gaggioli A (2018) Brain and virtual reality: What do they have in common and how to exploit their potential. Annu Rev Cyberther Telemed 16, 3-7.

[142] Muratore M, Tuena C, Pedroli E, Cipresso P, Riva G (2019) Virtual reality as a possible tool for the assessment of selfawareness. Front Behav Neurosci 13, 62. 